\title{
Hamiltonian Hopf bifurcation with symmetry
}

\author{
Pascal Chossat ${ }^{1}$, Juan-Pablo Ortega ${ }^{2}$, and Tudor S. Ratiu ${ }^{3}$
}

November 27, 2000

\begin{abstract}
In this paper we study the appearance of branches of relative periodic orbits in Hamiltonian Hopf bifurcation processes in the presence of compact symmetry groups that do not generically exist in the dissipative framework. The theoretical study is illustrated with several examples.
\end{abstract}

\section{Introduction}

Let $(V, \omega)$ be a symplectic vector space and $G$ be a compact Lie group acting linearly and symplectically on $V$. Let now be a one-parameter family of $G$-invariant Hamiltonians $h_{\lambda} \in$ $C^{\infty}(V)^{G}$ such that for each value of the parameter $\lambda$, the origin is an equilibrium of the associated Hamiltonian vector field, that is, $\mathbf{d} h_{\lambda}(0)=0$ for arbitrary $\lambda$. In this paper we will study the nonlinear implications of the following linear behavior: suppose that there is a value of the parameter $\lambda_{\circ}$ and a pair of eigenvalues $\pm i \nu_{\circ}$ in the spectrum of the linearization at zero of the dynamics induced by the Hamiltonian vector field $X_{h_{\lambda_{0}}}$ that behave as in Figure 1.1 when we move the parameter $\lambda$ around $\lambda_{\circ}$. Such a behavior in the parametrical motion of the eigenvalues is usually referred to as Hamiltonian Hopf bifurcation vdM85, denomination that we will use here, even though it also appears in the literature as $1:-1$ resonance, $1: 1$ non-semisimple resonance, and Krein collision. The reference to the Hopf bifurcation comes from the analogy with the codimension one non-conservative case in which a one-parameter family of vector fields has a pair of eigenvalues that cross the imaginary axis at a critical value of the parameter (the "classical" Hopf bifurcation). The case of $G$-equivariant vector fields ( $G$ compact) has led to the successful theory of Hopf bifurcation with symmetry which was initiated by GoS85 and which was exposed in its most achieved form in [Fi94 (see also ChL00 for a comprehensive exposition).

\footnotetext{
${ }^{1}$ Institut Nonlinéaire de Nice, UMR 129, CNRS-UNSA, 1361, route des Lucioles, 06560 Valbonne, France. chossat@inln.cnrs.fr.

${ }^{2}$ Département de Mathématiques, École Polytechnique Fédérale de Lausanne. CH-1015 Lausanne. Switzerland. Juan-Pablo.Ortega@epfl.ch.

${ }^{3}$ Department of Mathematics, University of California, Santa Cruz, Santa Cruz, CA 95064, USA, and Département de Mathématiques, École Polytechnique Fédérale de Lausanne. CH-1015 Lausanne. Switzerland. Tudor.Ratiu@epfl.ch. Research partially supported by NSF Grant DMS-9802378 and FNS Grant 21-54138.98.
} 


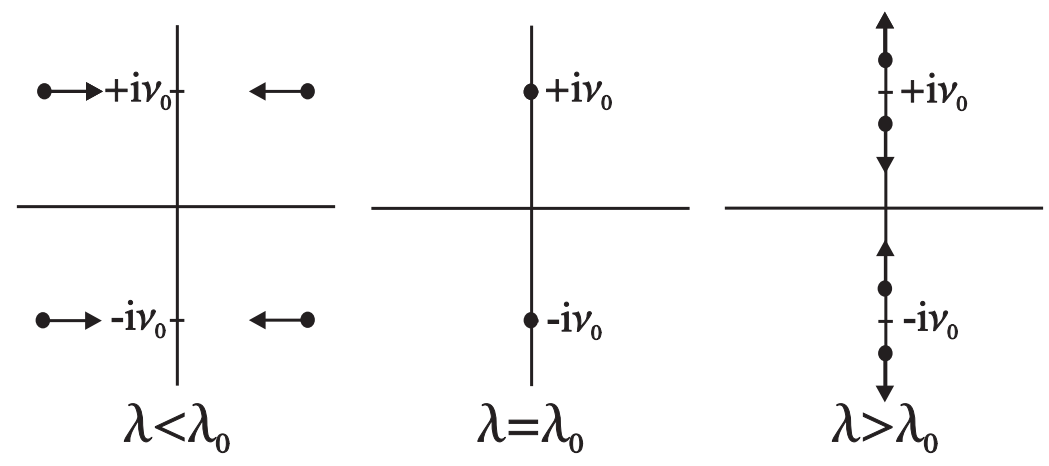

Figure 1.1: Motion of eigenvalues in a Hamiltonian Hopf bifurcation.

The history of the Hamiltonian Hopf bifurcation in the non-symmetric setup is very long and we shall not attempt to survey it here. We just refer to MeyS71, Mey86, vdM85, vdM96, Bri90, GMSD95 and references therein for discussions.

The only works that we know of dealing with the Hamiltonian symmetric case are vdM90 and [KMS96]. In the first paper it is shown that one can apply the non-symmetric results on Hamiltonian Hopf bifurcation on some of the fixed point spaces corresponding to isotropy subgroups of the symmetry of the system, provided that certain dimensional restrictions are fulfilled. [KMS96] studies branches of (stable) three-tori that can be obtained out of a Hamiltonian Hopf bifurcation process with a symmetry given by the semi-direct product of $D_{2}$ with $T^{2} \times S^{1}$. See also [Bri90a].

Natural dynamical elements that show up in the study of systems that present a continuous symmetry group $G$ are the so called relative equilibria $(\boldsymbol{R E})$ and relative periodic orbits $(\boldsymbol{R P O s})$, that is, motions that project onto equilibria and periodic orbits in the quotient space $V / G$, respectively. In our work we will see that whenever a Hopf-like motion of eigenvalues occurs in a Hamiltonian system with symmetry, one can prove the existence of periodic and relative periodic motions at the non linear level, for values of the parameter nearby $\lambda_{\circ}$, whose number we will estimate at each energy level. The existence of periodic motions, in the presence of some dimensional restrictions that we have eliminated, was something known to the above quoted authors. As to the relative periodic orbits, they are found in those papers only after a reduction has been performed that makes the problem equivalent to that of searching periodic orbits in the relevant quotient space. Since this reduction cannot always be carried out in a straightforward manner we will follow an approach in which the existence of relative periodic orbits is proved in the original space $V$.

Our approach to this problem will be based in the combined use of five tools:

(i) Reduction method of Vanderbauwhede and van der Meer VvdM95: it allows us to substitute the search of periodic and relative periodic orbits by the search of relative equilibria of a $S^{1}$-symmetric associated Hamiltonian system (usually referred to as normal form). 
(ii) Generic structure of the generalized eigenspaces corresponding to the colliding eigenvalues [DMM92]: it determines the most plausible reduced space in which we should work after applying (i).

(iii) Equivariant Williamson normal form [MD93]: it is used to normalize the linear term of the equation that defines the $S^{1}$-relative equilibria that we are looking for.

(iv) Lyapunov-Schmidt reduction of the finite dimensional equation that defines the $S^{1}$ relative equilibria and formulation of the problem in terms of a bifurcation equation of gradient nature CLOR99 with very specific equivariance properties.

(v) Solution of the bifurcation equation using either topological or analytical methods.

The paper is structured as follows:

- In Section 2 we briefly review the abovementioned tools, set the notation that will be used throughout the paper, and explain in detail the hypotheses under which we will work, along with their implications. The expert can skip this section and use it just as a glossary.

- Section 3 is devoted to Theorem 3.3, which provides a lower estimate on the number of periodic and relative periodic branches that bifurcate from the origin if there is a collision of eigenvalues as in Figure 1.1.

- In Section 4.1 we study a system of two nonlinearly coupled harmonic oscillators in the presence of a magnetic field, that will lead us to the consideration of the general case of the Hamiltonian Hopf bifurcation in the presence of a $O(2)$ symmetry. We will see that, in contrast with the dissipative case, the $O(2)$-symmetry in a Hamiltonian Hopf bifurcation process gives rise to the appearance of numerous relative periodic motions. This example will also show that, in general, the topological methods utilized in Theorem 3.3 are not powerful enough to detect all the periodic and relative periodic elements of a particular system with a given symmetry, that is, the generality of this result is paid with its lack of sharpness. This circumstance will motivate a more hands-on approach to the problem in Section 4.3, where we will see that under additional hypotheses on the group action, sharper general results can be formulated that give account of all the dynamical richness evidenced in the example in Section 4.1.

- In Section 4.4 we use the previous results to show the existence of RPOs in Hamiltonian Hopf phenomena with spheric symmetry.

- For the sake of the clarity in the exposition, the proofs of some of the technical results needed in the main theorem are relegated to an appendix (Section 5) at the end of the paper. 


\section{Preliminaries and setup}

All throughout this paper, our discussions will mostly take place in a finite dimensional symplectic vector space $(V, \omega)$ on which the compact Lie group $G$ acts linearly and canonically, that is, respecting the symplectic form $\omega$. We will be interested in a one-parameter family of $G$ equivariant Hamiltonian vector fields $X_{h_{\lambda}}$, induced by the family of $G$-invariant Hamiltonians $h_{\lambda} \in C^{\infty}(V)^{G}, \lambda \in \mathbb{R}$, such that:

(H1) $h_{\lambda}(0)=0$ and $\mathbf{d} h_{\lambda}(0)=0$ for all $\lambda$.

(H2) There is a value $\lambda_{\circ}$ of the parameter $\lambda$ for which the $G$-equivariant infinitesimally symplectic linear map $A_{\lambda_{\circ}}:=D_{V} X_{h_{\lambda_{\circ}}}(0)$ is non singular and has $\pm i \nu_{\circ}$ in its spectrum $\left(\nu_{\circ} \neq 0\right)$.

In the following paragraphs we introduce some tools and notations that will be used all throughout the paper.

The resonance space Let $(V, \omega)$ be a symplectic vector space. It is easy to show that there is a bijection between linear Hamiltonian vector fields on $(V, \omega)$ and quadratic forms on $V$. Indeed, if $A: V \rightarrow V$ is an infinitesimally symplectic linear map, that is, a linear Hamiltonian vector field on $(V, \omega)$, its corresponding Hamiltonian function is given by

$$
Q_{A}(v):=\frac{1}{2} \omega(A v, v), \quad \text { for any } v \in V \text {. }
$$

Also, since $A$ belongs to the symplectic Lie algebra $\mathfrak{s p}(V)$, it admits a unique JordanChevalley decomposition Hu72, VvdM95] of the form $A=A_{s}+A_{n}$, where $A_{s} \in \mathfrak{s p}(V)$ is semisimple (complex diagonalizable), $A_{n} \in \mathfrak{s p}(V)$ is nilpotent, and $\left[A_{s}, A_{n}\right]=0$. If $i \nu_{\circ}$ is one of the eigenvalues of $A \in \mathfrak{s p}(V)$ and $T_{\nu_{\circ}}:=\frac{2 \pi}{\nu_{\circ}}$, we define the resonance space $U_{\nu_{\circ}}$ of $A$ with primitive period $T_{\nu_{\circ}}$ as

$$
U_{\nu_{\circ}}:=\operatorname{ker}\left(e^{A_{s} T_{\nu_{\circ}}}-I\right) .
$$

The resonance space $U_{\nu_{\circ}}$ has the following properties (see Wil36, GoS87, VvdM95):

(i) $U_{\nu_{\circ}}$ is equal to the direct sum of the real generalized eigenspaces of $A$ corresponding to eigenvalues of the form $\pm i k \nu_{\circ}$, with $k \in \mathbb{N}^{*}$.

(ii) The pair $\left(U_{\nu_{0}},\left.\omega\right|_{U_{\nu}}\right)$ is a symplectic subspace of $(V, \omega)$.

(iii) The mapping $\left.\theta \in S^{1} \mapsto e^{\frac{\theta}{\nu_{0}} A_{s}}\right|_{U_{\nu_{0}}}$ generates a symplectic $S^{1}$-linear action on $\left(U_{\nu_{0}},\left.\omega\right|_{U_{\nu_{0}}}\right)$, whose associated equivariant momentum map will be denoted by $\mathbf{J}: U_{\nu_{\circ}} \rightarrow \operatorname{Lie}\left(S^{1}\right)^{*} \simeq \mathbb{R}$.

(iv) If $(V, \omega)$ is a symplectic representation space of the Lie group $G$ and the Hamiltonian vector field $A$ is $G$-equivariant (equivalently, the quadratic form $Q_{A}$ is $G$-invariant), 
then the symplectic resonance subspace $\left(U_{\nu_{\circ}},\left.\omega\right|_{U_{\nu_{\circ}}}\right)$ is also $G$-invariant (this follows from the uniqueness of the Jordan-Chevalley decomposition of $A$, which implies that if $A$ is $G$-equivariant, so is $\left.A_{s}\right)$. Moreover, the $S^{1}$ and $G$ actions on $\left(U_{\nu_{\circ}},\left.\omega\right|_{U_{\nu_{\circ}}}\right)$ commute, which therefore defines a symplectic linear action of $G \times S^{1}$ on $U_{\nu_{0}}$. See the Appendix (Section 5) for a sketch of the proof of some of these facts.

The normal form reduction vdM85, vdM90, VvdM95 Let $\left(V, \omega, h_{\lambda}\right)$ be a $\lambda$-parameter family $\left(\lambda \in \Lambda\right.$, where $\Lambda$ is a Banach space) of $G$-Hamiltonian systems such that $h_{\lambda_{0}}(0)=0$, $\mathbf{d} h_{\lambda_{\circ}}(0)=0$, and the $G$-equivariant infinitesimally symplectic linear map $A:=D X_{h_{\lambda_{0}}}(0)$ is non singular and has $\pm i \nu_{\circ}$ as eigenvalues. Let $\left(U_{\nu_{\circ}},\left.\omega\right|_{U_{\nu_{0}}}\right)$ be the resonance space of $A$ with primitive period $T_{\nu_{\circ}}$. For each $k \geq 0$ there are a $C^{k}$-mapping $\psi: U_{\nu_{\circ}} \times \Lambda \rightarrow V$ and a $C^{k+1_{-}}$ mapping $\widehat{h_{\lambda}}: U_{\nu_{\circ}} \times \Lambda \rightarrow \mathbb{R}$ such that $\psi(0, \lambda)=0$, for all $\lambda \in \Lambda, D_{U_{\nu}} \psi\left(0, \lambda_{\circ}\right)=\mathbb{I}_{U_{\nu_{\circ}}}$, and $\widehat{h_{\lambda}}$ is a $G \times S^{1}$-invariant function that coincides with $h_{\lambda}$ up to order $k+1$. The interest of normalization is given by the fact that one can prove [VvdM95, Theorem 3.2] that if we stay close enough to zero in $U_{\nu_{\circ}}$ and to $\lambda_{\circ} \in \Lambda$, then the $S^{1}$-relative equilibria of the $G \times S^{1}$-invariant Hamiltonian $\widehat{h_{\lambda}}$ are mapped by $\psi(\cdot, \lambda)$ to the set of periodic solutions of $\left(V, \omega, h_{\lambda}\right)$ in a neighborhood of $0 \in V$ with periods close to $T_{\nu_{0}}$. Hence, in our future discussion we will substitute the problem of searching periodic orbits for $\left(V, \omega, h_{\lambda}\right)$ by that of searching the $S^{1}$-relative equilibria of the $G \times S^{1}$-invariant family of Hamiltonian systems $\left(U_{\nu_{\circ}},\left.\omega\right|_{U_{\nu_{0}}}, \widehat{h_{\lambda}}\right)$, that will be referred to as the equivalent system. Note that the properties of $\psi$ imply that

$$
\mathcal{A}:=\left.A\right|_{U_{\nu_{\circ}}}=\left.D_{V} X_{h_{\lambda_{\circ}}}(0)\right|_{U_{\nu_{\circ}}}=D_{V} X_{\left.h_{\lambda_{\circ}}\right|_{U_{\nu_{\circ}}}}(0)=D_{V} X_{\widehat{h_{\lambda_{\circ}}}}(0) .
$$

Generic structure of the resonance space $U_{\nu_{\circ}}$ and canonical form of the symplectic pair $\left(\left.\omega\right|_{U_{\nu_{0}}}, \mathcal{A}\right)$ After the remarks previously made, we know that the resonance space $U_{\nu_{\circ}}$ is a $G \times S^{1}$-symplectic vector space. The decomposition of $U_{\nu_{\circ}}$ into $G \times S^{1}$-irreducible subspaces that can be generically expected when the eigenvalues behave parametrically as in Figure 1.1 has been studied in [DMM92], where the authors concluded (Proposition 6.1 (3)) that the only possibility is

$$
U_{\nu_{\circ}}=U_{1} \oplus U_{2},
$$

where $U_{1}$ and $U_{2}$ are complex dual irreducible subspaces of $U_{\nu_{\circ}}$ in the sense of MRS88, Theorem 2.1]. In all that follows we will assume that we are in this generic situation.

Once we know the decomposition (2.2) of $U_{\nu_{\circ}}$ into irreducibles, the equivariant version of the Williamson normal form of Melbourne and Dellnitz guarantees [MD93, Table 2] that there is a basis of the vector space $U_{\nu_{\circ}}$ in which the simultaneous matricial expressions of $\left.\omega\right|_{U_{\nu_{\mathrm{o}}}}$ and $\mathcal{A}$ are either

(i)

$$
\mathcal{A}=\left(\begin{array}{cc}
\nu_{\circ} \mathbb{J}_{2 n} & \mathbb{I}_{2 n} \\
\mathbf{0} & \nu_{\circ} \mathbb{J}_{2 n}
\end{array}\right) \quad \text { and }\left.\quad \omega\right|_{U_{\nu}}=\mathbb{J}_{4 n}, \quad \text { or }
$$


(ii)

$$
\mathcal{A}=\left(\begin{array}{cc}
\nu_{\circ} \mathbb{J}_{2 n} & \mathbb{I}_{2 n} \\
\mathbf{0} & \nu_{\circ} \mathbb{J}_{2 n}
\end{array}\right) \quad \text { and }\left.\quad \omega\right|_{U_{\nu}}=-\mathbb{J}_{4 n}
$$

where $2 n=\operatorname{dim} U_{1}=\operatorname{dim} U_{2}, \mathbb{I}_{2 n}$ is the $2 n$-dimensional identity matrix, and $\mathbb{J}_{2 n}$ is defined as

$$
\mathbb{J}_{2 n}=\left(\begin{array}{cc}
\mathbf{0} & -\mathbb{I}_{n} \\
\mathbb{I}_{n} & \mathbf{0}
\end{array}\right)
$$

Given that the treatment of cases (i) and (ii) is completely analogous, we will focus in all that follows in expression (2.3). Moreover, whenever our family of $G$-Hamiltonian systems falls into the generic situation described in this paragraph, we will say that it satisfies the condition (H3). For clarity and future reference we state this condition explicitely:

(H3) The resonance space $U_{\nu_{\circ}}$ corresponding to the eigenvalues $\pm i \nu_{\circ}$ splits into two complex dual $G \times S^{1}$-irreducible subspaces. This condition is generic.

Relative equilibria and critical points Being consistent with the notation previously introduced, let $\mathcal{A}=\mathcal{A}_{s}+\mathcal{A}_{n}$ be the Jordan-Chevalley decomposition of $\mathcal{A} \in \mathfrak{s p}_{G}\left(U_{\nu_{\circ}}\right)$. We will denote by $\mathbf{J}: U_{\nu_{\circ}} \rightarrow \operatorname{Lie}\left(S^{1}\right)^{*} \simeq \mathbb{R}$ the equivariant momentum map associated to the symplectic $S^{1}$-linear action defined by $(\theta, v) \mapsto e^{\frac{\theta}{\nu_{0}} \mathcal{A}_{s}} v, \theta \in S^{1}, v \in U_{\nu_{\circ}}$. Also, for any $\xi \in \operatorname{Lie}\left(S^{1}\right) \simeq \mathbb{R}$ and any $v \in U_{\nu_{\circ}}$, we will write $\mathbf{J}^{\xi}(v):=\mathbf{J}(v) \xi$. The linearity of the action implies that, for any $\xi \in \operatorname{Lie}\left(S^{1}\right) \simeq \mathbb{R}$ and any $v \in U_{\nu_{\circ}}$, the momentum map $\mathbf{J}$ is uniquely determined by the expression

$$
\mathbf{J}^{\xi}(v)=\left.\frac{1}{2} \omega\right|_{U_{\nu_{\circ}}}(\xi \cdot v, v)
$$

where the dot in $\xi \cdot v$ means the associated representation of the Lie algebra $\operatorname{Lie}\left(S^{1}\right)$ on $U_{\nu}$ through the $S^{1}$-action. More specifically,

$$
\mathbf{J}(v)=\left.\frac{1}{2 \nu_{\circ}} \omega\right|_{U_{\nu_{\circ}}}\left(\mathcal{A}_{s} v, v\right) .
$$

For future reference we note that this relation implies that

$$
\mathbf{d}^{2} \mathbf{J}(0)(v, w)=\left.\omega\right|_{U_{\nu_{\circ}}}\left(\mathcal{A}_{s} v, w\right), \quad \text { for any } \quad v, w \in U_{\nu_{\circ}},
$$

which in the basis used to write (2.3) admits the following matricial expression:

$$
\mathbf{d}^{2} \mathbf{J}(0)=\left(\begin{array}{cc}
\mathbf{0} & \mathbb{J}_{2 n} \\
-\mathbb{J}_{2 n} & \mathbf{0}
\end{array}\right)
$$


A very interesting feature of the Hamiltonian framework is that the search for relative equilibria reduces to the determination of the critical points of the so called augmented Hamiltonian AM78]. In the particular case that we are dealing with, this remark translates into saying that the equivalent system $\left(U_{\nu_{0}},\left.\omega\right|_{U_{\nu_{0}}}, \widehat{h_{\lambda}}\right)$ has a $S^{1}$-relative equilibrium at $v \in U_{\nu_{\circ}}$ (which represents a periodic orbit of the original system $\left(V, \omega, h_{\lambda}\right)$ with period near $\left.T_{\nu_{\mathrm{o}}}\right)$ if and only if there is an element $\xi \in \operatorname{Lie}\left(S^{1}\right)$ for which

$$
\mathbf{d}\left(\widehat{h_{\lambda}}-\mathbf{J}^{\xi}\right)(v)=0 .
$$

Whenever we find a pair $(v, \xi)$ that satisfies (2.7), we will say that $v$ is a relative equilibrium with velocity $\xi$.

Expression (2.7) can be written as a gradient equation, which will be exploited profusely in our subsequent discussion. Indeed, let $\langle\cdot, \cdot\rangle$ be a $G \times S^{1}$-invariant inner product on $U_{\nu_{\circ}}$ (always available by the compactness of $G \times S^{1}$ ). For any $v \in U_{\nu_{0}}$, we define the gradient $\nabla_{U_{\nu_{\mathrm{o}}}}\left(\widehat{h_{\lambda}}-\mathbf{J}^{\xi}\right)(v)$ as the unique element in $U_{\nu_{\mathrm{o}}}$, such that for $w \in U_{\nu_{\mathrm{o}}}$ arbitrary

$$
\mathbf{d}\left(\widehat{h_{\lambda}}-\mathbf{J}^{\xi}\right)(v) \cdot w=\left\langle\nabla_{U_{\nu_{0}}}\left(\widehat{h_{\lambda}}-\mathbf{J}^{\xi}\right)(v), w\right\rangle .
$$

Also for future reference, we recall that the linearization $A_{\lambda}=D_{V} X_{h_{\lambda}}(0)$ of $X_{h_{\lambda}}$ at $0 \in V$, is a linear $G$-equivariant Hamiltonian vector field with associated quadratic Hamiltonian function $Q_{\lambda}$ given by

$$
Q_{\lambda}(v)=\frac{1}{2} \mathbf{d}^{2} h_{\lambda}(0)(v, v)
$$

that is:

$$
\mathbf{i}_{A_{\lambda}} \omega=\mathbf{d} Q_{\lambda} .
$$

The restriction $\mathcal{A}$ of $A_{\lambda_{\circ}}$ to $U_{\nu_{\circ}}$ is of course also Hamiltonian but in this case, by (2.1), the associated quadratic Hamiltonian function can be expressed in terms of the Hessian at 0 of the equivalent Hamiltonian $\widehat{h_{\lambda_{\circ}}}$ associated to $h_{\lambda_{\circ}}$. Indeed,

$$
\left.\mathbf{i}_{\mathcal{A}} \omega\right|_{U_{\nu_{\circ}}}=\mathbf{d} \mathcal{Q}_{\lambda_{\circ}}
$$

where, for any $v \in U_{\nu_{0}}$,

$$
\mathcal{Q}_{\lambda_{\diamond}}(v)=\frac{1}{2} \mathbf{d}^{2} h_{\lambda_{\diamond}}(0)(v, v)=\frac{1}{2} \mathbf{d}^{2} \widehat{h_{\lambda_{\diamond}}}(0)(v, v) .
$$

If we write (2.8) using the basis that produced the canonical form (2.3), the equality (2.9) guarantees that

$$
\mathbf{d}^{2} \widehat{h_{\lambda_{\circ}}}(0)=\left.\mathbf{d}^{2} h_{\lambda_{\circ}}(0)\right|_{U_{\lambda_{\circ}}}=-\mathbb{J}_{4 n} \mathcal{A}=\left(\begin{array}{cc}
\mathbf{0} & \nu_{\circ} \mathbb{J}_{2 n} \\
-\nu_{\circ} \mathbb{J}_{2 n} & -\mathbb{I}_{2 n}
\end{array}\right)
$$


Invariant splitting of the resonance space $U_{\nu_{0}}$ Using expressions (2.5) and (2.10) we can immediately construct a very convenient splitting of the resonance space $U_{\nu_{\circ}}$ : let $L: U_{\nu_{\circ}} \rightarrow U_{\nu_{\circ}}$ be the linear map defined by $\langle L(v), w\rangle=\mathbf{d}^{2}\left(\widehat{h_{\lambda_{\circ}}}-\mathbf{J}^{\nu_{\circ}}\right)(0)(v, w)$, for any $v, w \in U_{\nu_{\circ}}$. Using expressions (2.5), (2.6), and (2.10) we can write, using the basis introduced in (2.3), that we will use in all that follows:

$$
L=\left(\begin{array}{cc}
\mathbf{0} & \mathbf{0} \\
\mathbf{0} & -\mathbb{I}_{2 n}
\end{array}\right)
$$

Since the linear map $L$ is $G \times S^{1}$-equivariant and self-adjoint we can split $U_{\nu_{\circ}}=V_{0} \oplus V_{1}$ as the direct sum of the two $G \times S^{1}$-invariant subspaces,

$$
V_{0}:=\operatorname{ker} L=\left\{\left(\begin{array}{c}
\mathbf{a} \\
\mathbf{0}
\end{array}\right) \mid \mathbf{a} \in \mathbb{R}^{2 n}\right\}, \quad V_{1}:=\operatorname{Im} L=\left\{\left(\begin{array}{c}
\mathbf{0} \\
\mathbf{b}
\end{array}\right) \mid \mathbf{b} \in \mathbb{R}^{2 n}\right\} .
$$

Since by hypothesis (H3) we are in the generic situation, the resonance space $U_{\nu_{\circ}}$ splits as the sum of two complex dual irreducible subspaces with respect to the $G \times S^{1}$-representation DMM92]. Given that by construction $V_{0}$ and $V_{1}$ are $G \times S^{1}$-invariant and have the same dimension, the $G \times S^{1}$-representations on $V_{0}$ and $V_{1}$ are necessarily complex irreducible. We describe more precisely the interplay between the decomposition $U_{\nu_{\circ}}=V_{0} \oplus V_{1}$ and the $G \times S^{1}$-action in the following lemma.

Lemma 2.1 In all the matricial statements bellow we assume the use of the basis of the canonical form (2.3).

(a) Let $g \in G \times S^{1}$ arbitrary and $v=v_{0}+v_{1} \in U_{\nu_{\circ}}$, with $v_{0} \in V_{0}$ and $v_{1} \in V_{1}$. Then, there exists a orthogonal matrix $A_{g}$ such that $\left[A_{g}, \mathbb{J}_{2 n}\right]=0$ and

$$
g \cdot v=\left(\begin{array}{cc}
A_{g} & \mathbf{0} \\
\mathbf{0} & A_{g}
\end{array}\right) \cdot\left(\begin{array}{l}
v_{0} \\
v_{1}
\end{array}\right) .
$$

(b) The inner product on $U_{\nu_{\circ}}$ that takes the Euclidean form when expressed in the coordinates corresponding to the basis used to write the canonical form (2.3) is $G \times S^{1}$-invariant.

Proof (a) The $G \times S^{1}$-invariance of the spaces $V_{0}$ and $V_{1}$ implies, for any $g \in G \times S^{1}$, the existence of two invertible matrices $A_{g}$ and $B_{g}$ such that

$$
g \cdot v=\left(\begin{array}{cc}
A_{g} & \mathbf{0} \\
\mathbf{0} & B_{g}
\end{array}\right) \cdot\left(\begin{array}{l}
v_{0} \\
v_{1}
\end{array}\right)
$$

for any $v=v_{0}+v_{1} \in U_{\nu_{0}}$, with $v_{0} \in V_{0}$ and $v_{1} \in V_{1}$. Given that by hypothesis the family of Hamiltonians $h_{\lambda}$ is $G$-invariant, the linearization $\mathcal{A}$ in (2.3) of the vector field $X_{h_{\lambda_{\circ}}}$ at the origin is necessarily $G \times S^{1}$-equivariant and, consequently

$$
\left(\begin{array}{cc}
A_{g} & \mathbf{0} \\
\mathbf{0} & B_{g}
\end{array}\right)\left(\begin{array}{cc}
\nu_{\circ} \mathbb{J}_{2 n} & \mathbb{I}_{2 n} \\
\mathbf{0} & \nu_{\circ} \mathbb{J}_{2 n}
\end{array}\right)=\left(\begin{array}{cc}
\nu_{\circ} \mathbb{J}_{2 n} & \mathbb{I}_{2 n} \\
\mathbf{0} & \nu_{\circ} \mathbb{J}_{2 n}
\end{array}\right)\left(\begin{array}{cc}
A_{g} & \mathbf{0} \\
\mathbf{0} & B_{g}
\end{array}\right)
$$


which implies that $A_{g}=B_{g}$ and that $A_{g} \mathbb{J}_{2 n}=\mathbb{J}_{2 n} A_{g}$, for any $g \in G \times S^{1}$. We now see that the matrices $A_{g}$ are orthogonal: given that the $G \times S^{1}$-action is canonical, we have that

$$
\left(\begin{array}{cc}
A_{g}^{T} & \mathbf{0} \\
\mathbf{0} & A_{g}^{T}
\end{array}\right)\left(\begin{array}{cc}
\mathbf{0} & -\mathbb{I}_{2 n} \\
\mathbb{I}_{2 n} & \mathbf{0}
\end{array}\right)\left(\begin{array}{cc}
A_{g} & \mathbf{0} \\
\mathbf{0} & A_{g}
\end{array}\right)=\left(\begin{array}{cc}
\mathbf{0} & -\mathbb{I}_{2 n} \\
\mathbb{I}_{2 n} & \mathbf{0}
\end{array}\right)
$$

This equality guarantees that $A_{g}^{T} A_{g}=A_{g} A_{g}^{T}=\mathbb{I}_{2 n}$, as required.

(b) Let $\langle\cdot, \cdot\rangle$ be the inner product in the statement, that is, for any $v, w \in U_{\nu_{\circ}}$ whose coordinates in the basis of (2.3) are $v=\left(v_{1}, \ldots, v_{4 n}\right)$ and $w=\left(w_{1}, \ldots, w_{4 n}\right)$ we have that $\langle v, w\rangle=$ $v_{1} w_{1}+\ldots+v_{4 n} w_{4 n}$. Notice that, also in this basis, the inner product $\langle\cdot, \cdot\rangle$ can be expressed as

$$
\langle v, w\rangle=\left.\omega\right|_{U_{\nu_{0}}}\left(\mathbb{J}_{4 n} v, w\right) .
$$

Before we proceed notice that expression (2.12) together with the orthogonality of $A_{g}$ implies that for any $v \in U_{\nu_{\circ}}$ and any $g \in G$ we have that $\mathbb{J}_{4 n} g \cdot v=g \cdot \mathbb{J}_{4 n} v$. If we put together this fact with the canonical character of this action we obtain the invariance of the inner product. Indeed, for $v, w \in U_{\nu_{\circ}}$ and $g \in G \times S^{1}$ arbitrary, we have that

$$
\langle g \cdot v, g \cdot w\rangle=\left.\omega\right|_{U_{\nu_{\mathrm{o}}}}\left(\mathbb{J}_{4 n} g \cdot v, g \cdot w\right)=\left.\omega\right|_{U_{\nu_{\mathrm{o}}}}\left(g \cdot \mathbb{J}_{4 n} v, g \cdot w\right)=\left.\omega\right|_{U_{\nu_{\mathrm{o}}}}\left(\mathbb{J}_{4 n} v, w\right)=\langle v, w\rangle,
$$

which concludes the proof.

Remark 2.2 In all our subsequent discussions we will use the inner product presented in the previous lemma and the basis of the canonical form (2.3).

The quadratic part of the Hamiltonian and a final generic hypothesis The complex irreducibility of the $G \times S^{1}$-action on $V_{0}$ implies [GSS88, Lemma 3.4] that if $\mathcal{P}_{G \times S^{1}}\left(V_{0}\right)$ denotes the ring of real $G \times S^{1}$-invariant polynomials on $V_{0}$, one can choose a basis $\left\{F_{1}, \ldots, F_{l}\right\}$ of $\mathcal{P}_{G \times S^{1}}\left(V_{0}, V_{0}\right)$, that is, the finite type $\mathcal{P}_{G \times S^{1}}\left(V_{0}\right)$-module of $G \times S^{1}$-equivariant polynomial mappings of $V_{0}$ into itself, such that

$$
\left\{\begin{array}{l}
F_{1}=\mathbb{I}_{2 n} \\
F_{2}=\mathbb{J}_{2 n} \\
\operatorname{deg} F_{k}>1 \quad \forall k>2 .
\end{array}\right.
$$

Analogously, one can choose a Hilbert basis $\left\{\theta_{1}, \ldots, \theta_{r}\right\}$ of the module $\mathcal{P}_{G \times S^{1}}\left(V_{0}\right)$, such that

$$
\left\{\begin{array}{l}
\theta_{1}(v)=\|v\|^{2} \\
\operatorname{deg} \theta_{k}>2 \quad \forall k>1
\end{array}\right.
$$

In particular, the $G \times S^{1}$-invariance of the Hamiltonians $\widehat{h_{\lambda}}$ of the equivalent system $\left(U_{\nu_{0}},\left.\omega\right|_{U_{\nu_{0}}}, \widehat{h_{\lambda}}\right)$ implies that for each $\lambda$, the second derivative $\mathbf{d}^{2} \widehat{h_{\lambda}}(0)$, considered as a linear map $\mathbf{d}^{2} \widehat{h_{\lambda}}(0)$ : 
$V_{0} \oplus V_{1} \rightarrow V_{0} \oplus V_{1}$ is $G \times S^{1}$-equivariant. At the same time, since it is a Hessian, it is symmetric and therefore there are functions $\sigma, \rho, \tau, \psi \in C^{\infty}(\mathbb{R})$ such that:

$$
\mathbf{d}^{2} \widehat{h_{\lambda}}(0)=\left(\begin{array}{cc}
\sigma(\lambda) \mathbb{I}_{2 n} & \tau(\lambda) \mathbb{I}_{2 n}+\psi(\lambda) \mathbb{I}_{2 n} \\
\tau(\lambda) \mathbb{I}_{2 n}-\psi(\lambda) \mathbb{J}_{2 n} & \rho(\lambda) \mathbb{I}_{2 n}
\end{array}\right)
$$

where, by (2.10), we have the following initial conditions: $\sigma\left(\lambda_{\circ}\right)=0, \rho\left(\lambda_{\circ}\right)=-1, \tau\left(\lambda_{\circ}\right)=0$, and $\psi\left(\lambda_{\circ}\right)=\nu_{\circ}$. In all that follows we will assume the following generic hypothesis:

(H4) The one-parameter family of $G$-Hamiltonian systems $\left(V, \omega, h_{\lambda}\right)$ satisfies that $\sigma^{\prime}\left(\lambda_{\circ}\right) \neq 0$, where $\sigma(\lambda) \in C^{\infty}(\mathbb{R})$ is the smooth real function introduced in (2.15).

The previous generic hypothesis that will be of much technical importance in what follows is related, as we show in the following lemma, to the motion of eigenvalues depicted in Figure 1.1.

Lemma 2.3 Let $\left(V, \omega, h_{\lambda}\right)$ be a one-parameter family of $G$-Hamiltonian systems satisfying hypotheses (H1) through (H4). Then, there is a part of the spectrum of the linearization of the Hamiltonian vector fields $X_{h_{\lambda}}$ at zero that behaves as in Figure 1.1 as we move the parameter $\lambda$.

Proof We first compute the eigenvalues of $\mathcal{A}_{\lambda}$, that is, the restriction of $A_{\lambda}$ to the symplectic subspace $\left(U_{\nu_{\circ}},\left.\omega\right|_{U_{\nu_{0}}}\right)$. This vector field is Hamiltonian. More specifically:

$$
\left.\mathbf{i}_{\mathcal{A}_{\lambda}} \omega\right|_{U_{\nu_{\circ}}}=\mathbf{d} \mathcal{Q}_{\lambda}
$$

where, for any $v \in U_{\nu_{0}}$,

$$
\mathcal{Q}_{\lambda}(v)=\frac{1}{2} \mathbf{d}^{2} h_{\lambda}(0)(v, v)=\frac{1}{2} \mathbf{d}^{2} \widehat{h_{\lambda}}(0)(v, v) .
$$

If we use in (2.17) the specific form for the symplectic form $\omega$ introduced in (2.3) we obtain that:

$$
\mathcal{A}_{\lambda}=\mathbb{J}_{4 n} \mathbf{d}^{2} \widehat{h_{\lambda}}(0)
$$

We now use (2.15) and obtain that:

$$
\mathcal{A}_{\lambda}=\left(\begin{array}{cc}
-\tau(\lambda) \mathbb{I}_{2 n}+\psi(\lambda) \mathbb{J}_{2 n} & -\rho(\lambda) \mathbb{I}_{2 n} \\
\sigma(\lambda) \mathbb{I}_{2 n} & \tau(\lambda) \mathbb{I}_{2 n}+\psi(\lambda) \mathbb{J}_{2 n}
\end{array}\right) .
$$

We compute the eigenvalues of this matrix using the well-known fact Hal74, p. 102, ex. 9] that if $A, B, C$, and $D$ commute, then

$$
\operatorname{det}\left(\begin{array}{cc}
A & B \\
C & D
\end{array}\right)=\operatorname{det}(A D-B C) \text {. }
$$


Indeed, using this relation, we see that the characteristic polynomial of $\mathcal{A}_{\lambda}$ is

$$
\begin{aligned}
\operatorname{det}\left(\mathcal{A}_{\lambda}-\mu \mathbb{I}_{4 n}\right)=\left(\begin{array}{cc}
(-\tau(\lambda)-\mu) \mathbb{I}_{2 n}+\psi(\lambda) \mathbb{J}_{2 n} & -\rho(\lambda) \mathbb{I}_{2 n} \\
\sigma(\lambda) \mathbb{I}_{2 n} & (\tau(\lambda)-\mu) \mathbb{I}_{2 n}+\psi(\lambda) \mathbb{J}_{2 n}
\end{array}\right) \\
=\operatorname{det}\left[\left(\mu^{2}-\tau(\lambda)^{2}-\psi(\lambda)^{2}+\rho(\lambda) \sigma(\lambda)\right) \mathbb{I}_{2 n}-2 \mu \psi(\lambda) \mathbb{I}_{2 n}\right] \\
=\operatorname{det}\left(\begin{array}{cc}
\left(\mu^{2}-\tau(\lambda)^{2}-\psi(\lambda)^{2}+\rho(\lambda) \sigma(\lambda)\right) \mathbb{I}_{n} & 2 \mu \psi(\lambda) \mathbb{I}_{n} \\
-2 \mu \psi(\lambda) \mathbb{I}_{n} & \left(\mu^{2}-\tau(\lambda)^{2}-\psi(\lambda)^{2}+\rho(\lambda) \sigma(\lambda)\right) \mathbb{I}_{n}
\end{array}\right) \\
=\operatorname{det}\left[\left(\left(\mu^{2}-\tau(\lambda)^{2}-\psi(\lambda)^{2}+\rho(\lambda) \sigma(\lambda)\right)^{2}+(2 \mu \psi(\lambda))^{2}\right) \mathbb{I}_{n}\right] \\
=\left(\left(\left(\mu^{2}-\tau(\lambda)^{2}-\psi(\lambda)^{2}+\rho(\lambda) \sigma(\lambda)\right)^{2}+4 \mu^{2} \psi(\lambda)^{2}\right)^{n} .\right.
\end{aligned}
$$

Consequently, the eigenvalues of $\mathcal{A}_{\lambda}$ are

$$
\mu(\lambda)= \pm \sqrt{\tau(\lambda)^{2}-\rho(\lambda) \sigma(\lambda)-\psi(\lambda)^{2} \pm 2|\psi(\lambda)| \sqrt{\rho(\lambda) \sigma(\lambda)-\tau(\lambda)^{2}}} .
$$

We are now in position to show that hypothesis (H4) implies the motion depicted in Figure 1.1. Indeed, assume that $\sigma^{\prime}\left(\lambda_{\circ}\right) \neq 0$. Let $f_{1}(\lambda)=\rho(\lambda) \sigma(\lambda)-\tau(\lambda)^{2}$. Note that $f_{1}\left(\lambda_{\circ}\right)=0$ and $f_{1}^{\prime}\left(\lambda_{\circ}\right)=-\sigma^{\prime}\left(\lambda_{\circ}\right)$, that by hypothesis is different from zero. This implies that the function $f_{1}$ changes sign at $\lambda_{\circ}$. More explicitely, suppose that $\sigma^{\prime}\left(\lambda_{\circ}\right)<0$ (the case $\sigma^{\prime}\left(\lambda_{\circ}\right)>0$ is completely analogous); in that case $f_{1}(\lambda)<0$ for $\lambda<\lambda_{\circ}$ and $f_{1}(\lambda)>0$ for $\lambda>\lambda_{\circ}$. Since we have that:

$$
\mu(\lambda)= \pm \sqrt{-f_{1}(\lambda)-\psi(\lambda)^{2} \pm 2|\psi(\lambda)| \sqrt{f_{1}(\lambda)}}= \pm \sqrt{-\left(\sqrt{f_{1}(\lambda)} \pm|\psi(\lambda)|\right)^{2}} .
$$

then, by considering the cases $\lambda\left\langle\lambda_{\circ}, \lambda=\lambda_{\circ}\right.$, and $\lambda>\lambda_{\circ}$ in the previous expression taking into account the changes of sign in $f_{1}$, we obtain the evolution of eigenvalues illustrated in Figure 1.1.

Remark 2.4 The generic hypothesis (H4) is a sufficient but not necessary condition for obtaining a behavior of the eigenvalues as in Figure 1.1, that is, such an evolution can take place even for systems in which $\sigma^{\prime}\left(\lambda_{\circ}\right)=0$.

\section{Hamiltonian Hopf bifurcation and relative periodic orbits}

The main goal of this section is the statement and proof of a result that will provide and estimate on the number of relative periodic orbits of a one-parameter family of $G$-Hamiltonian systems $\left(V, \omega, h_{\lambda}\right)$ that satisfies the hypotheses $(\mathbf{H 1})$ through $(\mathbf{H} 4)$, formulated in the previous section.

We will begin by introducing some classical definitions that will make more explicit some of the concepts used in the previous paragraphs.

It appears very frequently in examples dealing with symmetric families of Hamiltonian systems that the canonical symmetry group $G$ contains a continuous globally Hamiltonian 
symmetry: suppose that $G$ contains a Lie subgroup $H$ of positive dimension. We say that the canonical action of $H$ on $V$ is globally Hamiltonian when we can associate to it an equivariant momentum map $\mathbf{K}: V \rightarrow \mathfrak{h}^{*}$ which is defined by the fact that its components $\mathbf{K}^{\xi}:=\langle\mathbf{K}, \xi\rangle \in$ $C^{\infty}(\mathbb{R}), \xi \in \mathfrak{h}$, have as associated Hamiltonian vector fields the infinitesimal generators of the action

$$
\xi_{V}(v)=\left.\frac{d}{d t}\right|_{t=0} \exp t \xi \cdot v \quad \xi \in \mathfrak{h}, v \in V
$$

Definition 3.1 Let $(V, \omega, h)$ be a Hamiltonian system with a symmetry given by the canonical action of the Lie group $H$ on $V$. The point $v \in V$ is called a relative periodic point (RPP), if there is a $\tau>0$ and an element $g \in H$ such that

$$
F_{t+\tau}(v)=g \cdot F_{t}(v) \quad \text { for any } \quad t \in \mathbb{R},
$$

where $F_{t}$ is the flow of the Hamiltonian vector field $X_{h}$. The set

$$
\gamma(v):=\left\{F_{t}(v) \mid t>0\right\}
$$

is called a relative periodic orbit (RPO) through $v$. The constant $\tau>0$ is its relative period and the group element $g \in H$ is its phase shift.

Proposition 3.2 Let $(V, \omega, h)$ be a Hamiltonian system with a globally Hamiltonian symmetry given by the canonical action of the Lie group $H$ on $V$ with associated momentum map $\mathbf{K}$ : $V \rightarrow \mathfrak{h}^{*}$. If the Hamiltonian vector field $X_{h-\mathbf{K}^{\xi}}, \xi \in \mathfrak{h}$, has a periodic point $v \in V$ with period $\tau$, then the point $v$ is a RPP of $X_{h}$ with relative period $\tau$ and phase shift $\exp \tau \xi$.

Proof Let $F_{t}$ be the flow of the Hamiltonian vector field $X_{h}$ and $K_{t}(v)=\exp t \xi \cdot v$ that of $X_{\mathbf{K}}$. By Noether's Theorem:

$$
\left[X_{h}, X_{\mathbf{K}^{\xi}}\right]=-X_{\left\{h, \mathbf{K}^{\xi}\right\}}=0,
$$

where the bracket $\{\cdot, \cdot\}$ denotes the Poisson bracket associated to the symplectic form $\omega$. Due to this equality, we can write (see for instance AMR99, Corollary 4.1.27]) the following expression for $G_{t}$, the flow of $X_{h-\mathbf{K}^{\xi}}$ :

$$
G_{t}(v)=\lim _{n \rightarrow \infty}\left(F_{t / n} \circ K_{-t / n}\right)^{n}(v)=\left(K_{-t} \circ F_{t}\right)(v)=\exp -t \xi \cdot F_{t}(v) .
$$

Since by hypothesis the point $v$ is periodic for $G_{t}$ with period $\tau$, we have that

$$
v=\exp -\tau \xi \cdot F_{\tau}(v)
$$

or, equivalently,

$$
F_{\tau}(v)=\exp \tau \xi \cdot v,
$$


as required.

Using the previous proposition, we will reduce the search for RPOs of a generic oneparameter family of $G$-Hamiltonian systems $\left(V, \omega, h_{\lambda}\right)$ that satisfies conditions (H1), (H2), (H3), and (H4), to the search for periodic orbits of the vector fields of the form $X_{h_{\lambda}-\mathbf{K}^{\xi}}$, and will prove the following result:

Theorem 3.3 Let $\left(V, \omega, h_{\lambda}\right)$ be a one-parameter family of $G$-Hamiltonian systems that satisfies conditions $(\mathbf{H 1}),(\mathbf{H 2}),(\mathbf{H 3})$, and $\mathbf{( H 4 )}$. Suppose that $G$ contains a Lie subgroup $H$ of positive dimension with associated equivariant momentum map $\mathbf{K}: V \rightarrow \mathfrak{h}^{*}$. Let $U_{\nu_{\circ}}$ be the resonance space with primitive period $T_{\nu_{0}}$. Then, for each $\xi \in \mathfrak{h}$ whose norm $\|\xi\|$ is small enough, there are at least, in each energy level nearby zero and for each value of the parameter $\lambda$ near $\lambda_{\circ}$, as many relative periodic orbits as the number of equilibria of a $G^{\xi} \times S^{1}$-equivariant vector field defined on the unit sphere on $V_{0}$. The symbol $G^{\xi}$ denotes the adjoint isotropy subgroup of the element $\xi \in \mathfrak{h}$, that is,

$$
G^{\xi}=\left\{g \in G \mid \operatorname{Ad}_{g} \xi=\xi\right\}
$$

Remark 3.4 If we are just interested in looking for purely periodic orbits it suffices to use Theorem 3.3 with $\xi=0$. Conversely, if we use this result with a value of the parameter $\xi \neq 0$ we cannot conclude that the predicted RPOs are not trivial, that is, that they are not just periodic orbits. This point will become much clearer in the examples presented in the following sections.

Remark 3.5 In terms of practical applications, the relevance of Theorem 3.3 is given by the fact that the estimate that it provides in terms of the number of equilibria of an equivariant vector field on the sphere can sometimes be calculated via topological arguments, as we will see later on.

Proof We will work in the basis of the resonance space $U_{\nu_{\circ}}$ provided by the equivariant Williamson normal form, in particular we will use the matricial expressions (2.3), which are consistent with the decomposition $U_{\nu_{\circ}}=V_{0} \oplus V_{1}$ presented in (2.11). Recall that the subspaces $V_{0}$ and $V_{1}$ are $G \times S^{1}$-invariant. Abusing the notation a little bit we will use the symbol $\xi$ to denote both an element of the Lie algebra $\mathfrak{h} \subset \mathfrak{g}$ and its representation on $V_{0}$ and $V_{1}$. Using Lemma 2.1 we can write, for each $v=v_{0}+v_{1} \in U_{\nu^{\circ}}$ represented in the previously mentioned basis,

$$
\xi_{U_{\nu_{\mathrm{o}}}}(v)=\xi \cdot v_{0}+\xi \cdot v_{1}=\left(\begin{array}{cc}
\xi & \mathbf{0} \\
\mathbf{0} & \xi
\end{array}\right)\left(\begin{array}{l}
v_{0} \\
v_{1}
\end{array}\right)
$$

Note that, also by Lemma 2.1, that the matrix $\xi$ is skew-symmetric, $\xi^{T}=-\xi$, therefore normal, and hence diagonalizable. The same Lemma implies that the linear map $\xi: V_{0} \rightarrow V_{0}$ associated 
to $\xi \in \mathfrak{h}$ commutes with $\mathbb{J}_{2 n},\left[\xi, \mathbb{J}_{2 n}\right]=0$, and consequently these two endomorphisms can be simultaneously diagonalized.

We recall that,

$$
\langle\mathbf{K}(v), \xi\rangle=\frac{1}{2} \omega(\xi \cdot v, v)=\frac{1}{2}\left(v_{0}, v_{1}\right)\left(\begin{array}{rr}
\mathbf{0} & \xi \\
-\xi & \mathbf{0}
\end{array}\right)\left(\begin{array}{l}
v_{0} \\
v_{1}
\end{array}\right)
$$

In particular,

$$
\mathbf{d}^{2} \mathbf{K}^{\xi}(0)=\left(\begin{array}{rr}
\mathbf{0} & \xi \\
-\xi & \mathbf{0}
\end{array}\right)
$$

We start the proof by defining the $\mathbb{R} \times \mathfrak{h}$-parameter family of Hamiltonian functions given by

$$
h_{\lambda, \xi}=h_{\lambda}-\mathbf{K}^{\xi}
$$

Due to the hypotheses on the family $h_{\lambda}$, the quadratic nature of the momentum map $\mathbf{K}$, and the fact that $h_{\lambda, 0}=h_{\lambda}$, the family $h_{\lambda, \xi}$ satisfies the hypotheses of the Normal Form Reduction Theorem VvdM95. Therefore, a new family $\widehat{h_{\lambda, \xi}}$ can be constructed such that, for any value $(\lambda, \xi)$ of the parameters, the Hamiltonian $\widehat{h_{\lambda, \xi}}$ is $S^{1}$-invariant with respect to the action generated by the semisimple part of the linearization at zero of $X_{h_{\lambda_{0}, 0}}=X_{h_{\lambda_{0}}}$, that is, $(\theta, v) \mapsto \mathrm{e}^{\frac{\theta}{\nu_{0}} \mathcal{A}^{s}} v, \theta \in S^{1}$, with

$$
\mathcal{A}^{s}=\left(\begin{array}{cc}
\nu_{\circ} \mathbb{J}_{2 n} & \mathbf{0} \\
\mathbf{0} & \nu_{\circ} \mathbb{J}_{2 n}
\end{array}\right)
$$

The Normal Form Reduction Theorem guarantees that the $S^{1}$-relative equilibria of $\widehat{h_{\lambda, \xi}}$ are in correspondence with the periodic orbits $h_{\lambda, \xi}$ which, by Proposition 3.2, are RPOs of $h_{\lambda}$. The quadratic nature of the momentum map $\mathbf{K}$ and its $S^{1}$-invariance imply that $\widehat{h_{\lambda, \xi}}$ can be chosen to be of the form

$$
\widehat{h_{\lambda, \xi}}=\widehat{h_{\lambda}}-\mathbf{K}^{\xi}
$$

with $\widehat{h_{\lambda}}$ the normal form for the family $h_{\lambda}$.

As a result of these premises, the RPOs that we are looking for will be given by the critical points of the function $\widehat{h_{\lambda}}-\mathbf{K}^{\xi}-\mathbf{J}^{\zeta+\alpha}$, that is, the elements $(v, \alpha, \lambda, \xi) \in U_{\nu_{\circ}} \times \mathbb{R} \times \mathbb{R} \times \mathfrak{h}$ for which the function

$$
F^{\zeta}(v, \alpha, \lambda):=\nabla_{U_{\nu_{\circ}}}\left(\widehat{h_{\lambda}}-\mathbf{K}^{\xi}-\mathbf{J}^{\zeta+\alpha}\right)(v)
$$

has a zero. As customary, the gradient in the previous expression is constructed using the inner product introduced in Lemma 2.1. 
Lyapunov-Schmidt reduction and the bifurcation equation The linearization $L^{\zeta}$ : $U_{\nu_{\circ}} \rightarrow U_{\nu_{\circ}}$ of the equation (3.2) at the point $\left(0,0, \lambda_{\circ}, 0\right)$ produces, in the usual basis, the expression:

$$
L^{\zeta}=\mathbf{d}^{2}\left(\widehat{h_{\lambda}}-\mathbf{J}^{\zeta}\right)(0)=\mathbf{d}^{2}\left(h_{\lambda}-\mathbf{J}^{\zeta}\right)(0)=\left(\begin{array}{cc}
\mathbf{0} & \left(1-\frac{\zeta}{\nu_{\circ}}\right) \nu_{\circ} \mathbb{J}_{2 n} \\
-\left(1-\frac{\zeta}{\nu_{\circ}}\right) \nu_{\circ} \mathbb{J}_{2 n} & -\mathbb{I}_{2 n}
\end{array}\right) .
$$

By looking at this matricial expression we see that it is possible to Lyapunov-Schmidt reduce the bifurcation problem posed in (3.2) whenever $\zeta=\nu_{0}$, which we will assume in the sequel. In those circumstances ker $L^{\nu_{\circ}}=V_{0}, \operatorname{Im} L^{\nu_{\circ}}=V_{1}$. Let $\mathbb{P}: U_{\nu_{\circ}} \rightarrow V_{0}$ be the $G \times S^{1}$-equivariant projection associated to the splitting $U_{\nu_{\circ}}=V_{0} \oplus V_{1}$. The equation $(\mathbb{I}-\mathbb{P}) F^{\nu_{\circ}}\left(v_{0}+v_{1}, \alpha, \lambda, \xi\right)=$ $(\mathbb{I}-\mathbb{P}) \nabla_{U_{\nu_{\circ}}}\left(\widehat{h_{\lambda}}-\mathbf{K}^{\xi}-\mathbf{J}^{\nu_{\circ}+\alpha}\right)\left(v_{0}+v_{1}\right)=0$ defines, via the Implicit Function Theorem, a function $v_{1}: V_{0} \times \mathbb{R} \times \mathbb{R} \times \mathfrak{h} \rightarrow V_{1}$, such that

$(\mathbb{I}-\mathbb{P}) F^{\nu_{\circ}}\left(v_{0}+v_{1}\left(v_{0}, \alpha, \lambda, \xi\right), \alpha, \lambda, \xi\right)=(\mathbb{I}-\mathbb{P}) \nabla_{U_{\nu_{\circ}}}\left(\widehat{h_{\lambda}}-\mathbf{K}^{\xi}-\mathbf{J}^{\nu_{\circ}+\alpha}\right)\left(v_{0}+v_{1}\left(v_{0}, \alpha, \lambda, \xi\right)\right)=0$.

Notice that the function $v_{1}\left(v_{0}, \alpha, \lambda, \xi\right)$ is $G^{\xi} \times S^{1}$-equivariant, since this is the symmetry under which $F^{\nu}$ is equivariant, that is, for any $g \in G^{\xi} \times S^{1}$, we have that $v_{1}\left(g \cdot v_{0}, \alpha, \lambda, \xi\right)=$ $g \cdot v_{1}\left(v_{0}, \alpha, \lambda, \xi\right)$.

The final Lyapunov-Schmidt $G^{\xi} \times S^{1}$-equivariant reduced bifurcation equation, whose zeros provide us with the RPOs that we are after, is given by $B: V_{0} \times \mathbb{R} \times \mathbb{R} \times \mathfrak{h} \rightarrow V_{0}$, where

$$
\begin{aligned}
B\left(v_{0}, \alpha, \lambda, \xi\right) & =\mathbb{P} F^{\nu_{\circ}}\left(v_{0}+v_{1}\left(v_{0}, \alpha, \lambda, \xi\right), \alpha, \lambda, \xi\right)=\mathbb{P} \nabla_{U_{\nu_{\circ}}}\left(\widehat{h_{\lambda}}-\mathbf{K}^{\xi}-\mathbf{J}^{\nu_{\circ}+\alpha}\right)\left(v_{0}+v_{1}\left(v_{0}, \alpha, \lambda, \xi\right)\right) \\
& =\nabla_{U_{\nu_{\circ}}}\left(\widehat{h_{\lambda}}-\mathbf{K}^{\xi}-\mathbf{J}^{\nu_{\circ}+\alpha}\right)\left(v_{0}+v_{1}\left(v_{0}, \alpha, \lambda, \xi\right)\right) \quad(\text { by }(3.4)) .
\end{aligned}
$$

We collect the main properties of the reduced bifurcation equation in the following

Lemma 3.6 The reduced bifurcation equation (3.5) is $G^{\xi} \times S^{1}$-equivariant with respect to the action of this Lie group on $V_{0}$ and it is the gradient of $a G^{\xi} \times S^{1}$-invariant function defined on $V_{0}$, that is,

$$
B\left(v_{0}, \alpha, \lambda, \xi\right)=\nabla_{V_{0}} g\left(v_{0}, \alpha, \lambda, \xi\right),
$$

where the function $g: V_{0} \times \operatorname{Lie}\left(S^{1}\right) \times \mathbb{R} \times \mathfrak{h} \rightarrow V_{0}$ is defined by

$$
g\left(v_{0}, \alpha, \lambda, \xi\right)=\left(\widehat{h_{\lambda}}-\mathbf{J}^{\nu_{\circ}+\alpha}-\mathbf{K}^{\xi}\right)\left(v_{0}+v_{1}\left(v_{0}, \alpha, \lambda, \xi\right)\right) .
$$

Proof The $G^{\xi} \times S^{1}$-equivariance is a direct consequence of the construction of $B$. As to the gradient character of $B$, note first that for any $w \in V_{1}$ we have that

$$
\begin{aligned}
\left\langle F^{\nu_{\circ}}\left(v_{0}+v_{1}\left(v_{0}, \alpha, \lambda, \xi\right), \alpha, \lambda, \xi\right), w\right\rangle & =\left\langle F^{\nu_{\circ}}\left(v_{0}+v_{1}\left(v_{0}, \alpha, \lambda, \xi\right), \alpha, \lambda, \xi\right),(\mathbb{I}-\mathbb{P}) w\right\rangle \\
& =\left\langle(\mathbb{I}-\mathbb{P}) F^{\nu_{\circ}}\left(v_{0}+v_{1}\left(v_{0}, \alpha, \lambda, \xi\right), \alpha, \lambda, \xi\right), w\right\rangle=0
\end{aligned}
$$


where the last equality follows from the construction of the function $v_{1}$ through expression (3.4). Now, let $u \in V_{0}$ arbitrary. We write:

$$
\begin{aligned}
\left\langle B\left(v_{0}, \alpha, \lambda, \xi\right), u\right\rangle & =\left\langle\mathbb{P} F^{\nu_{\circ}}\left(v_{0}+v_{1}\left(v_{0}, \alpha, \lambda, \xi\right), \alpha, \lambda, \xi\right), u\right\rangle \\
& =\left\langle F^{\nu_{\circ}}\left(v_{0}+v_{1}\left(v_{0}, \alpha, \lambda, \xi\right), \alpha, \lambda, \xi\right), u\right\rangle \\
& =\left\langle F^{\nu_{\circ}}\left(v_{0}+v_{1}\left(v_{0}, \alpha, \lambda, \xi\right), \alpha, \lambda, \xi\right), u+D_{V_{0}} v_{1}\left(v_{0}, \alpha, \lambda, \xi\right) \cdot u\right\rangle \quad \text { (by (3.6)) } \\
& =\left\langle\nabla_{U_{\nu_{0}}}\left(\widehat{h_{\lambda}}-\mathbf{J}^{\nu_{\circ}+\alpha}-\mathbf{K}^{\xi}\right)\left(v_{0}+v_{1}\left(v_{0}, \alpha, \lambda, \xi\right)\right), u+D_{V_{0}} v_{1}\left(v_{0}, \alpha, \lambda, \xi\right) \cdot u\right\rangle \\
& =\mathbf{d}\left(\widehat{h_{\lambda}}-\mathbf{J}^{\nu_{\circ}+\alpha}-\mathbf{K}^{\xi}\right)\left(v_{0}+v_{1}\left(v_{0}, \alpha, \lambda, \xi\right)\right) \cdot\left(u+D_{V_{0}} v_{1}\left(v_{0}, \alpha, \lambda, \xi\right) \cdot u\right) \\
& =\mathbf{d} g\left(v_{0}, \alpha, \lambda, \xi\right) \cdot u=\left\langle\nabla_{V_{0}} g\left(v_{0}, \alpha, \lambda, \xi\right), u\right\rangle,
\end{aligned}
$$

as required. This construction is a particular case of the one carried out in GMSD95] and [CLOR99.

Notational simplification: In order to make notation a little bit lighter we will assume in the rest of the proof, without loss of generality, that the system has been scaled in such a way that $\nu_{\circ}=1$ and $\lambda_{\circ}=0$.

The following lemmas provide a local description of the reduced bifurcation equation that will be much needed.

Lemma 3.7 The function $v_{1}$ introduced in (3.4) has the following two properties:

$$
\begin{aligned}
& \text { (i) } v_{1}(0, \alpha, \lambda, \xi)=0 \quad \text { for all } \quad \alpha, \lambda \in \mathbb{R} \text {, and } \xi \in \mathfrak{h} . \\
& \text { (ii) } D_{V_{0}} v_{1}(0, \alpha, \lambda, \xi)=-\frac{\tau(\lambda)}{\rho(\lambda)} \mathbb{I}_{2 n}-\frac{(1+\alpha)-\psi(\lambda)}{\rho(\lambda)} \mathbb{J}_{2 n}-\frac{1}{\rho(\lambda)} \xi
\end{aligned}
$$

Proof Part (i) is a consequence of the uniqueness of the solutions provided by the Implicit Function Theorem. The proof of part (ii) is supplied in the Appendix, Section 5.2.

The proof of the following lemma is a lengthy but straightforward computation.

Lemma 3.8 Let $B\left(v_{0}, \alpha, \lambda, \xi\right)$ be the reduced bifurcation equation, then:

(i)

$$
D_{V_{0}} B(0, \alpha, \lambda, \xi)=\frac{\sigma(\lambda) \rho(\lambda)-\tau^{2}(\lambda)-((1+\alpha)-\psi(\lambda))^{2}}{\rho(\lambda)} \mathbb{I}_{2 n}+\frac{2[(1+\alpha)-\psi(\lambda)]}{\rho(\lambda)} \mathbb{J}_{2 n} \xi+\frac{\xi^{2}}{\rho(\lambda)} .
$$

(ii) The principal part of the reduced bifurcation equation is given by the expression:

$$
\begin{aligned}
B\left(v_{0}, \alpha, \lambda, \xi\right)=\left(\lambda \sigma^{\prime}(0)+\alpha^{2}\right) v_{0}-\xi^{2} v_{0}-2 & \alpha \mathbb{J}_{2 n} \xi v_{0}-2 \psi^{\prime}(0) \alpha \lambda v_{0} \\
& +2 \psi^{\prime}(0) \lambda \mathbb{J}_{2 n} \xi v_{0}+C\left(v_{0}^{(3)}\right)+\text { h.o.t. }
\end{aligned}
$$

where $C\left(v_{0}^{(3)}\right)$ is the trilinear operator obtained by taking the gradient of the fourth order term in the $v_{0}$-expansion of $\widehat{h_{\lambda_{\circ}}}\left(v_{0}+v_{1}\left(v_{0}, 0,0,0\right)\right)$. 
We now write the reduced bifurcation equation in polar coordinates, that is, we define

$$
B_{p}\left(r, u_{0}, \alpha, \lambda, \xi\right)=B\left(r u_{0}, \alpha, \lambda, \xi\right),
$$

where $r \in \mathbb{R}$ and $u_{0} \in S^{\operatorname{dim} V_{0}-1}$. We introduce the function

$$
F\left(r, u_{0}, \alpha, \lambda, \xi\right)=\frac{\left\langle B\left(r u_{0}, \alpha, \lambda, \xi\right), u_{0}\right\rangle}{r} .
$$

By looking at (3.9) it is clear that the function $F$ is smooth at the origin, $F(0,0,0,0,0)=0$ and that $D_{\lambda} F(0,0,0,0,0)=\sigma^{\prime}(0) \neq 0$, by hypothesis $(\mathbf{H 4})$. Therefore, the Implicit Function Theorem guarantees the existence of a smooth function $\lambda\left(r, u_{0}, \alpha, \xi\right)$ such that $\lambda(0,0,0,0)=0$ and $F\left(r, u_{0}, \alpha, \lambda\left(r, u_{0}, \alpha, \xi\right), \xi\right)=0$. This equality implies that if we substitute the function $\lambda\left(r, u_{0}, \alpha, \xi\right)$ on the reduced bifurcation equation, this time considered as a vector field on $V_{0}$, we obtain a new $(\alpha, \xi)$-parameter dependent vector field

$$
G\left(r, u_{0}, \alpha, \xi\right)=B_{b}\left(r, u_{0}, \alpha, \lambda\left(r, u_{0}, \alpha, \xi\right), \xi\right)
$$

which due to the fact that $\left\langle B_{b}\left(r, u_{0}, \alpha, \lambda\left(r, u_{0}, \alpha, \xi\right), \xi\right), u_{0}\right\rangle=0$ is, for each small enough fixed value of $r$, a $G^{\xi} \times S^{1}$-equivariant vector field on the sphere on $V_{0}$ of radius $r$, whose zeroes constitute solutions of the reduced bifurcation equation.

Method for the optimal use of Theorem 3.3 The optimal and most organized way to apply Theorem 3.3 consists of using the estimate it provides in the fixed point subspaces $V_{0}^{H}$ corresponding to the various subgroups $H$ in the lattice of isotropy subgroups of the $G \times S^{1}$ action on $V_{0}$, replacing the group $G \times S^{1}$ by $N(H)$, which is a group that acts on $V_{0}^{H}$ (not necessarily in an irreducible manner). The symbol $N(H)$ denotes the normalizer of $H$ in $G \times S^{1}$ and $V_{0}^{H}$ is the vector subspace of $V_{0}$ formed by the vectors fixed by $H$. We make more explicit this comment in the following paragraphs.

Let $H$ be a subgroup of $G \times S^{1}$. If $\pi: G \times S^{1} \rightarrow G$ denotes the canonical projection and $\pi(H)=: K \subset G$, Proposition 7.2 in GSS88 guarantees the existence of a group homomorphism $\theta: K \rightarrow S^{1}$ such that

$$
H=\left\{(k, \theta(k)) \in G \times S^{1} \mid k \in K\right\} .
$$

In our discussion we will be concerned with spatiotemporal symmetries, that is, subgroups $H$ of $G \times S^{1}$ for which the homomorphism $\theta: K \rightarrow S^{1}$ is nontrivial. Using the characterization (3.11) it is straightforward to see that

$$
N(H)=N_{G}(K) \times S^{1} .
$$

The $N_{G}(K)$-action on $U_{\nu_{\circ}}^{H}$ is globally Hamiltonian with momentum map $\mathbf{K}^{H}: U_{\nu_{\circ}}^{H} \rightarrow \operatorname{Lie}\left(N_{G}(K)\right)^{*}$ given by the restriction of the $G$-momentum map to $U_{\nu_{\circ}}^{H}$, that is, for any $v \in U_{\nu_{\circ}}^{H}$ and any $\xi \in \operatorname{Lie}\left(N_{G}(K)\right)$, we have that

$$
\left\langle\mathbf{K}^{H}(v), \xi\right\rangle=\langle\mathbf{K}(v), \xi\rangle .
$$

The same statement applies to the $S^{1}$-action. Using these objects we can reformulate Theorem 3.3 on the fixed point spaces $V_{0}^{H}$. 
Corollary 3.9 Let $\left(V, \omega, h_{\lambda}\right)$ be a one-parameter family of $G$-Hamiltonian systems that satisfies conditions (H1), (H2), (H3), and (H4). Let $H$ be a spatiotemporal isotropy subgroup of the $G \times S^{1}$-action on $V_{0}$, such that $\operatorname{dim} V_{0}^{H}=2 k$ and $K:=\pi(H)$. Then, for each $\xi \in \operatorname{Lie}\left(N_{G}(K)\right)$ whose norm $\|\xi\|$ is small enough, there are at least in each energy level nearby zero and for each value of the parameter $\lambda$ near $\lambda_{\circ}$, as many relative periodic orbits as the number of equilibria of a $N_{G}(K)^{\xi} \times S^{1}$-equivariant vector field on the unit sphere on $V_{0}^{H}$. The relative periods of these RPOs are close to $T_{\nu_{\circ}}$, and their phase shifts are close to $\exp T_{\nu_{0}} \xi$. The symbol $N_{G}(K)^{\xi}$ denotes the adjoint isotropy subgroup of the element $\xi \in \operatorname{Lie}\left(N_{G}(K)\right)$, that is,

$$
N_{G}(K)^{\xi}=\left\{g \in N_{G}(K) \mid \operatorname{Ad}_{g} \xi=\xi\right\} .
$$

The mapping $\pi: G \times S^{1} \rightarrow G$ denotes the canonical projection.

As we already said, both the previous result and Theorem 3.3 can be used to look for purely periodic motions by taking in their respective statements $\xi=0$. There is a situation of special interest:

Periodic orbits with maximal isotropy subgroup: Let $H$ be a maximal isotropy subgroup of the $G \times S^{1}$-action on $V_{0}$. In the presence of maximality we have at our disposal the following convenient result:

Lemma 3.10 Let $H$ be a maximal isotropy subgroup of the compact $G \times S^{1}$-action on $V_{0}$. Let $N$ be the Lie group $N(H) / H$ and $N^{0}$ be the connected component of the identity of $N$. Then either

(i) $N^{0} \simeq S^{1}$, and $N / N^{0}=\{I d\}$ or $N / N^{0} \simeq \mathbb{Z}_{2}$, or

(ii) $N^{0} \simeq S U(2)$ and $N \simeq S U(2)$.

In the first case we say that $H$ is a maximal complex subgroup. In the second case we say that $H$ a maximal quaternionic subgroup.

Proof It is a straightforward combination of the general result for linear actions of compact Lie groups Bre72, G83, GSS88] with Proposition 12.5 in [GoSt85 that eliminates the possibility of having real maximal isotropy subgroups when the compact group in question is $G \times S^{1}$.

Using the previous lemma and an additional genericity hypothesis, the estimate given in Theorem 3.3 can be made very explicit:

Corollary 3.11 Let $\left(V, \omega, h_{\lambda}\right)$ be a generic one-parameter family of $G$-Hamiltonian systems that satisfies conditions $(\mathbf{H 1}),(\mathbf{H 2}),(\mathbf{H 3})$, and $\mathbf{( H 4 )}$. Let $H$ be a maximal isotropy subgroup of the $G \times S^{1}$-action on $V_{0}$ such that $\operatorname{dim}\left(V_{0}^{H}\right)=l \neq 0$. Then: 
(i) If $N^{0} \simeq S^{1}$ there are at least $l / 2$ (if $N / N^{0}=\{$ Id $\}$ ) or $l / 4$ (if $N / N^{0} \simeq \mathbb{Z}_{2}$ ) branches of periodic solutions with isotropy $H$ coming out of the origin as one varies the parameter $\lambda$, with periods close to $T_{\nu_{\mathrm{o}}}$.

(ii) If $N^{0} \simeq S U(2)$ there are at least $l / 4$ branches of periodic solutions with isotropy $H$ coming out of the origin as one varies the parameter $\lambda$, with periods close to $T_{\nu_{0}}$.

Proof We will adapt to our problem the approach followed in CKM95, Koe95 for rotating waves. The main idea behind the proof consists of using the maximality hypothesis to give a numerical evaluation of the estimate in Corollary 3.9, that is, the number of equilibria of a $N(H) / H$-equivariant vector field on the sphere $S^{l-1}$.

More especifically, let $G^{H}:=\left.G\right|_{V_{0}^{H}}$ be the restriction of the vector field $G$ on $V_{0}$, defined in (3.10), to the fixed point set $V_{0}^{H}$, and $G_{r}^{H}\left(u_{0}, \alpha\right):=G^{H}\left(r, u_{0}, \alpha\right)$ be the $N$-equivariant vector field on $S_{r}^{l-1}$ obtained by fixing $r$ in the mapping $G^{H}$ (note that in our case $\xi=0$ since we are looking for periodic orbits). The zeroes of this vector field are in one to one correspondence with the solutions that we search. Due to the maximality hypothesis on the subgroup $H$, the $N$-action on the sphere $S_{r}^{l-1}$ is free and therefore the corresponding orbit space $S_{r}^{l-1} / N$ is a smooth manifold onto which we can project the $N$-equivariant vector field $G_{r}^{H}$. Let $\bar{G}_{r}^{H}$ be the projected vector field. Due to the genericity hypothesis in the statement, the Poincaré-Hopf Theorem allows us to say that $\bar{G}_{r}^{H}$ has at least $\chi\left(S_{r}^{l-1} / N\right)$ equilibria, where $\chi$ denotes the Euler characteristic. These zeroes lift to equilibria of the restriction of the reduced bifurcation equation to $V_{0}^{H}$, due to the gradient character (see Lemma 3.6) of $B$ and consequently of its restriction to $V_{0}^{H}$.

In order to conclude our argument it is enough to show that $\chi\left(S^{l-1} / N\right)$ corresponds to the estimates provided in the statement of the theorem. In the first case, when $N^{0} \simeq S^{1}$, the dimension of $V_{0}^{H}$ is necessarily even (we will write $l=2 k$ for certain $k \in \mathbb{N}$ ) and there are two possibilities: the quotient $N / N^{0}$ is either $\{I d\}$ or it is isomorphic to $\mathbb{Z}_{2}$. If $N / N^{0}=\{I d\}$ :

$$
\chi\left(S^{l-1} / N\right)=\chi\left(\left(S^{l-1} / N^{0}\right) /\left(N / N^{0}\right)\right)=\chi\left(S^{2 k-1} / S^{1}\right)=\chi\left(\mathbb{C P}^{k-1}\right)=k=\frac{l}{2} .
$$

If $N / N^{0} \simeq \mathbb{Z}_{2}$ :

$$
\chi\left(S^{l-1} / N\right)=\chi\left(\left(S^{l-1} / N^{0}\right) /\left(N / N^{0}\right)\right)=\chi\left(\mathbb{C P}^{k-1} / \mathbb{Z}_{2}\right)=\frac{k}{2}=\frac{l}{4},
$$

where we used the well-known fact that if $G$ is a finite group acting freely on a manifold $M$, then (see for instance [Kaw91, Corollary 5.22])

$$
\chi\left(\frac{M}{G}\right)=\frac{\chi(M)}{|G|} .
$$

Finally, if $H$ is maximal quaternionic then $l=\operatorname{dim}\left(V_{0}^{H}\right)=4 k$ for some $k \in \mathbb{N}$, necessarily, and

$$
\chi\left(S^{l-1} / N\right)=\chi\left(S^{4 k-1} / S U(2)\right)=\chi\left(\mathbb{H} \mathbb{P}^{k-1}\right)=k=\frac{l}{4} .
$$


The calculation of the Euler characteristic $\chi\left(\mathbb{H} \mathbb{P}^{k-1}\right)$ of the quaternionic projective space is made using an argument based the spectral series of Leray (see for instance [BT82]).

The computations that we just carried out give us periodic orbits for a fixed $r$. Moving smoothly this parameter we obtain the branches required in the statement of the theorem.

\section{Bifurcation of non-periodic relative periodic orbits in the presence of extra hypotheses}

The tools presented in Theorem 3.3 for the search of RPOs based on topological methods produce estimates that, as we will see in the following examples, have some limitations, in particular we have no example where it guarantees the bifurcation of non periodic RPOs. This circumstance has motivated us to use a more analytical approach under dimensional hypotheses that are satisfied in very relevant situations. A detailed study of the bifurcation equation in the presence of these hypotheses will provide us with sharper estimates that completely describe all the bifurcation phenomena that we see in the examples.

\subsection{Motivating example: two coupled harmonic oscillators subjected to a magnetic field as an example of symmetric Hamiltonian Hopf bifurcation}

We consider the system formed by two identical particles with unit charge in the plane, subjected to identical harmonic forces, to a homogeneous magnetic field perpendicular in direction to the plane of motion, and to an interaction potential that will preserve certain group of symmetry. We will denote by $\left(q_{1}, q_{2}\right)$ the coordinates of the configuration space of the first particle and by $\left(q_{3}, q_{4}\right)$ those of the second one. If $\gamma$ is a constant that determines the intensity of the magnetic field, it is easy to see that the Hamiltonian function of the system described above is

$$
\begin{aligned}
H(\mathbf{q}, \mathbf{p})=\frac{1}{2 m}\left(p_{1}^{2}+p_{2}^{2}+p_{3}^{2}+p_{4}^{2}\right) & +\left(\frac{\gamma^{2}}{2 m}-\frac{k}{2}\right)\left(q_{1}^{2}+q_{2}^{2}+q_{3}^{2}+q_{4}^{2}\right) \\
& +\frac{\gamma}{m}\left(p_{1} q_{2}-p_{2} q_{1}\right)+\frac{\gamma}{m}\left(p_{3} q_{4}-p_{4} q_{3}\right)+f\left(\pi_{1}^{i}, \pi_{2}^{i}, \pi_{3}^{i}\right),
\end{aligned}
$$

where

$$
\pi_{1}^{i}=q_{i}^{2}+q_{i+2}^{2}, \quad \pi_{2}^{i}=p_{i}^{2}+p_{i+2}^{2}, \quad \pi_{3}^{i}=p_{i} q_{i+2}-p_{i+2} q_{i}, \quad \pi_{4}^{i}=q_{i} p_{i}+q_{i+2} p_{i+2}, \quad i \in\{1,2\},
$$

and $f$ is a higher order function on its variables that expresses a non linear interaction between the two particles.

This system has, for all values of the parameters $\gamma$ and $k$, an equilibrium at the point $\left(q_{1}, q_{3}, q_{2}, q_{4}, p_{1}, p_{3}, p_{2}, p_{4}\right)=(\mathbf{0}, \mathbf{0})$. The linearization of the dynamics at that point is represented by the matrix (the coordinates are ordered as in the previous equality)

$$
\mathcal{A}_{k}=\left(\begin{array}{cc}
-\frac{\gamma}{m} \mathbb{J}_{4} & \frac{1}{m} \mathbb{I}_{4} \\
\left(k-\frac{\gamma^{2}}{m}\right) \mathbb{I}_{4} & -\frac{\gamma}{m} \mathbb{J}_{4}
\end{array}\right),
$$


whose eigenvalues are

$$
\lambda_{k}= \pm \frac{1}{m} \sqrt{k m-2 \gamma^{2} \pm 2 \gamma \sqrt{\gamma^{2}-k m}}
$$

If we move the parameter $k$ around the value $k_{\circ}=\gamma^{2} / m$ these eigenvalues present a Hamiltonian Hopf behavior like the one depicted in Figure 1.1.

We now study the symmetries of the system. Note that after the assumptions on the interaction function $f$, the system is invariant under the canonical $S^{1}$-action given by the lifted action to the phase space of

$$
(\varphi, \mathbf{q}) \longmapsto\left(\begin{array}{cccc}
\cos \varphi & -\sin \varphi & 0 \\
\sin \varphi & \cos \varphi & & 0 \\
0 & \cos \varphi & -\sin \varphi \\
0 & & \sin \varphi & \cos \varphi
\end{array}\right) \cdot \mathbf{q},
$$

where $\mathbf{q}=\left(q_{1}, q_{3}, q_{2}, q_{4}\right)$, and by the transformation

$$
\tau \cdot\left(\begin{array}{c}
q_{1} \\
q_{2} \\
q_{3} \\
q_{4}
\end{array}\right)=\left(\begin{array}{c}
q_{1} \\
q_{2} \\
-q_{3} \\
-q_{4}
\end{array}\right)
$$

The momentum map $\mathbf{K}: \mathbb{R}^{8} \rightarrow \mathbb{R}$ associated to the $S^{1}$-action is given by the expression $\mathbf{K}(\mathbf{q}, \mathbf{p})=p_{3} q_{1}-q_{3} p_{1}-p_{2} q_{4}+p_{4} q_{2}$.

If we now look at the linearization (4.2) evaluated at the Hopf value $k_{\circ}=\gamma^{2} / \mathrm{m}$ we see that in this case $V_{0}$ consists of the points of the form $\left(q_{1}, q_{3}, q_{2}, q_{4}, \mathbf{0}\right)$. The $S^{1}$-action on $V_{0}$ generated by the semisimple part of $\mathcal{A}_{k_{\circ}}$ can be written as

$$
(\theta, \mathbf{q}) \longmapsto \mathrm{e}^{-\theta \frac{\gamma}{m} \mathbb{J}_{4}} \cdot \mathbf{q} .
$$

In order to better study the group actions on $V_{0}$ we will perform a linear change of variables. Let $\left(z_{1}, z_{2}, \overline{z_{1}}, \overline{z_{2}}\right)$ be the new (complex) coordinates, given by

$$
\begin{aligned}
& z_{1}=q_{1}+q_{4}+i q_{2}-i q_{3} \\
& z_{2}=q_{1}-q_{4}+i q_{2}+i q_{3} \\
& \overline{z_{1}}=q_{1}+q_{4}-i q_{2}+i q_{3} \\
& \overline{z_{2}}=q_{1}-q_{4}-i q_{2}-i q_{3} .
\end{aligned}
$$

If we take as new angles $\psi_{1}$ and $\psi_{2}$, defined by:

$$
\psi_{1}=\varphi+\frac{\gamma}{m} \theta, \quad \psi_{2}=\varphi-\frac{\gamma}{m} \theta,
$$

we realize that the previously introduced actions form a $O(2) \times S^{1}$-action on $V_{0}$ that takes the following convenient simple expression:

$$
\left(\psi_{1}, \psi_{2}\right) \cdot\left(z_{1}, z_{2}\right)=\left(\mathrm{e}^{i \psi_{1}} z_{1}, \mathrm{e}^{i \psi_{2}} z_{2}\right) \quad \text { and } \quad \tau \cdot\left(z_{1}, z_{2}\right)=\left(z_{2}, z_{1}\right) .
$$


That is, we have shown that the system of two coupled harmonic oscillators subjected to a magnetic field, whose Hamiltonian is given by (4.1) can be taken as an example of Hamiltonian Hopf bifurcation with $O(2) \times S^{1}$-symmetry, which we will study in full generality in the following subsection.

\subsection{RPOs in Hamiltonian Hopf bifurcation with $O(2)$-symmetry}

Having the example in the previous section as a motivation we will study in what follows the RPOs that appear in a Hamiltonian Hopf bifurcation phenomenon in the presence of a $O(2)-$ symmetry. The simplicity of this symmetry will allow us to explicitly write down the principal part of the reduced bifurcation equation in full generality, and to read off directly from it the RPOs that we are looking for.

We start by recalling that in the canonical coordinates introduced in (2.3) the principal part of the reduced bifurcation equation is, by Lemma 3.8, equal in our case to

$$
\begin{aligned}
B\left(v_{0}, \alpha, \lambda, \xi\right)=\left(\lambda \sigma^{\prime}\left(\lambda_{\circ}\right)+\alpha^{2} \nu_{\circ}^{2}\right) v_{0}-\xi^{2} v_{0}- & 2 \alpha \nu_{\circ} \mathbb{J}_{4} \xi v_{0}-2 \psi^{\prime}\left(\lambda_{\circ}\right) \nu_{\circ} \alpha \lambda v_{0} \\
& +2 \psi^{\prime}\left(\lambda_{\circ}\right) \lambda \mathbb{J}_{4} \xi v_{0}+\mathbb{P}^{4} h_{\lambda_{\circ}}(0)\left(v_{0}^{(4)}\right)+\text { h.o.t. }
\end{aligned}
$$

We now rewrite this expression in the coordinates $\left(z_{1}, z_{2}, \overline{z_{1}}, \overline{z_{2}}\right)$ in which the $O(2) \times S^{1}$-action looks like (4.4). In doing so we need to express in these new coordinates the matrix $\mathbb{J}_{4} \xi$, which can be easily achieved by using the explicit expression of the change of variables (4.3). Indeed, we have that in those coordinates

$$
\mathbb{J}_{4} \xi \equiv\left(\begin{array}{cccc}
1 & 0 & 0 & 0 \\
0 & -1 & 0 & 0 \\
0 & 0 & 1 & 0 \\
0 & 0 & 0 & -1
\end{array}\right)
$$

and therefore, the first terms of the expansion of the reduced bifurcation equation are:

$$
B(\mathbf{z}, \alpha, \lambda, \xi)=\left(\lambda \sigma^{\prime}\left(\lambda_{\circ}\right)+\alpha^{2} \nu_{\circ}^{2}\right)\left(\begin{array}{c}
z_{1} \\
z_{2} \\
\overline{z_{1}} \\
\overline{z_{2}}
\end{array}\right)-2 \nu_{\circ} \alpha \xi\left(\begin{array}{c}
z_{1} \\
-z_{2} \\
\overline{z_{1}} \\
-\overline{z_{2}}
\end{array}\right)+\left(\begin{array}{c}
\left(a\left|z_{1}\right|^{2}+b\left|z_{2}\right|^{2}\right) z_{1} \\
\left(a\left|z_{2}\right|^{2}+b\left|z_{1}\right|^{2}\right) z_{2} \\
\left(a\left|z_{1}\right|^{2}+b\left|z_{2}\right|^{2}\right) \overline{z_{1}} \\
\left(a\left|z_{2}\right|^{2}+b\left|z_{1}\right|^{2}\right) \overline{z_{2}}
\end{array}\right)+\ldots
$$

where the coefficients $a$ and $b$ are related to the fourth order terms in the expansion of the Hamiltonian, that is, $\mathbb{P} \mathbf{d}^{4} h_{\lambda_{\circ}}(0)\left(v_{0}^{(4)}\right)$. In order to keep the simplicity of the exposition we will assume that these two coefficients are non zero and non equal (otherwise we would have to go to higher orders in expression (4.5)). The RPOs that we are looking for are given by the solutions of the system of equations:

$$
\begin{aligned}
& 0=\left(\lambda \sigma^{\prime}\left(\lambda_{\circ}\right)+\alpha^{2} \nu_{\circ}^{2}\right) z_{1}-2 \nu_{\circ} \alpha \xi z_{1}+\left(a\left|z_{1}\right|^{2}+b\left|z_{2}\right|^{2}\right) z_{1}+\ldots \\
& 0=\left(\lambda \sigma^{\prime}\left(\lambda_{\circ}\right)+\alpha^{2} \nu_{\circ}^{2}\right) z_{2}+2 \nu_{\circ} \alpha \xi z_{2}+\left(a\left|z_{2}\right|^{2}+b\left|z_{1}\right|^{2}\right) z_{2}+\ldots
\end{aligned}
$$


Since $\sigma^{\prime}\left(\lambda_{\circ}\right) \neq 0$, equation (4.6) can be easily solved by dividing the expression by $z_{1}$ and then using the Implicit Function Theorem to define a function

$$
\lambda \equiv \lambda\left(z_{1}, z_{2}, \alpha, \xi\right)=\frac{1}{\sigma^{\prime}\left(\lambda_{\circ}\right)}\left[-\alpha^{2} \nu_{\circ}^{2}+2 \nu_{\circ} \alpha \xi-\left(a\left|z_{1}\right|^{2}+b\left|z_{2}\right|^{2}\right)+\ldots\right]
$$

that substituted into (4.6) solves it. Hence, plugging (4.8) into (4.7) we reduce the problem to solving a scalar equation which can be done again via the Implicit Function Theorem: we divide (4.6) by $z_{1}$ and (4.7) by $z_{2}$ and obtain:

$$
0=4 \nu_{\circ} \alpha \xi+(a-b)\left(\left|z_{2}\right|^{2}-\left|z_{1}\right|^{2}\right)+\ldots
$$

Since for equivariance reasons $z_{1}$ and $z_{2}$ always appear in the tail of the previous expression as combinations of $\left|z_{1}\right|^{2}$ and $\left|z_{2}\right|^{2}$, the hypotheses on the coefficients $a$ and $b$ allow us to solve this final scalar equation by defining a function

$$
\left|z_{2}\right|^{2} \equiv\left|z_{2}\right|^{2}\left(\left|z_{1}\right|^{2}, \alpha, \xi\right)=\left|z_{1}\right|^{2}-\frac{4 \nu_{\circ} \alpha \xi}{a-b}+\ldots
$$

As we illustrate in Figure 4.1, the solution (4.10) predicts, for each fixed value of the norm

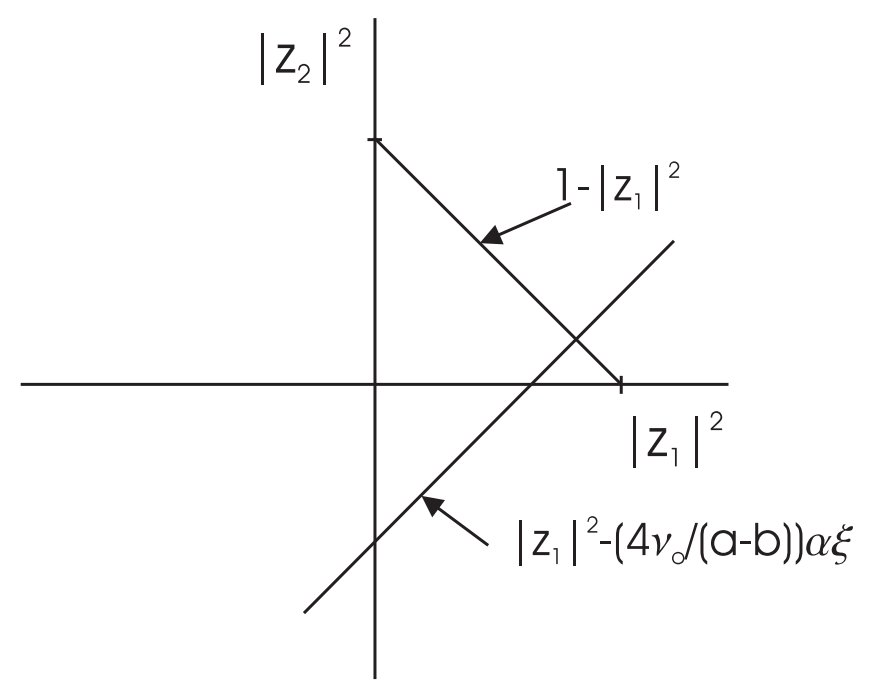

Figure 4.1: Parameterization of the RPOs in the Hamiltonian Hopf bifurcation with $O(2)-$ symmetry.

$\left|z_{1}\right|^{2}+\left|z_{2}\right|^{2}$ a one-parameter family of RPOs that are obtained by making vary the product $\alpha \xi$. More explicitly, and using Figure 4.1, suppose that $\left|z_{1}\right|^{2}+\left|z_{2}\right|^{2}=1$ and that the value $\alpha \xi$ is fixed, then, the intersection of the lines $\left|z_{2}\right|^{2}=1-\left|z_{1}\right|^{2}$ and $\left|z_{2}\right|^{2}=\left|z_{1}\right|^{2}-\frac{4 \nu_{\circ} \alpha \xi}{a-b}$ provides us with the abovementioned RPO. 
Notice that all these RPOs cannot be predicted by merely using Theorem 3.3 since even though all the hypotheses needed in this result are fulfilled, it only predicts two RPOs for each value of the norm $\left|z_{1}\right|^{2}+\left|z_{2}\right|^{2}$. Moreover, one cannot be sure that these are not just periodic motions since the predicted orbits could lie in fixed spaces of maximal isotropy, thereby implying their periodicity.

Remark 4.1 In contrast with the Hamiltonian case, the Hopf bifurcation of nontrivial RPOs in the dissipative case with $O(2)$ symmetry is subjected to the presence of a cubic order degeneracy in the normal form. Unfolding this singularity leads to a codimension two bifurcation problem where the RPOs appear as a secondary branching from the primary branches of periodic orbits.

\subsection{Hamiltonian Hopf bifurcation of RPOs for reduced integrable systems}

The analysis performed in the previous section dealing with the Hamiltonian Hopf bifurcation with $O(2)$-symmetry is a particular case of a more general situation. Indeed, the main feature in that example that allowed us to carry out a by hand in-depth study of the reduced bifurcation equation was the coincidence of one half the dimension of the reduced space $V_{0}$ with the dimension of the symmetry group $O(2) \times S^{1}$. We will see in this section that whenever we are in the presence of this reduced integrability hypothesis an analysis in the same style can be performed.

More explicitly, all along this section we will be dealing with $\left(V, \omega, h_{\lambda}\right)$, a one-parameter family of $G$-Hamiltonian systems that satisfies conditions (H1), (H2), (H3), and (H4) such that if $4 n$ is the dimension of the resonance space $U_{\nu_{\circ}}$ with primitive period $T_{\nu_{\circ}}$, then the rank of $G \times S^{1}$ equals $n$, that is, the maximal tori of the Lie group $G \times S^{1}$ have all dimension equal to $n$.

Let $\mathbb{T}^{n-1} \subset G$ be a maximal torus of $G$, and let $\xi \in \mathfrak{t}^{n-1}$ be an element in the Lie algebra of $\mathbb{T}^{n-1}$. Like in the previous section we can find coordinates in which the action of $\mathbb{T}^{n-1} \times S^{1}$ looks simple. Namely, there exists a set of complex coordinates $\left(z_{1}, \ldots, z_{n}, \overline{z_{1}}, \ldots, \overline{z_{n}}\right.$ ) (and conjugates) for $V_{0}$ and a set of angular coordinates $\left(\xi_{1}, \ldots, \xi_{n-1}\right)$ for the torus $T^{n-1}$, for which the $\mathbb{T}^{n-1}$-action looks like

$$
\left(\mathrm{e}^{i \xi_{1}}, \ldots, \mathrm{e}^{i \xi_{n-1}}\right) \cdot\left(z_{1}, \ldots, z_{n}\right)=\left(\mathrm{e}^{i \xi_{1}} z_{1}, \ldots, \mathrm{e}^{i \xi_{n-1}} z_{n-1}, \mathrm{e}^{i\left(c_{1} \xi_{1}+\ldots+c_{n-1} \xi_{n-1}\right)} z_{n}\right),
$$

where the coefficients $c_{1}, \ldots, c_{n-1}$ are rational constants. If we incorporate the $S^{1}$-action using these complex coordinates, the $\mathbb{T}^{n-1} \times S^{1}$-action looks like

$$
\left(\mathrm{e}^{i x i_{1}}, \ldots, \mathrm{e}^{i \xi_{n-1}}, \mathrm{e}^{i \alpha}\right) \cdot\left(z_{1}, \ldots, z_{n}\right)=\left(\mathrm{e}^{i\left(\xi_{1}+\alpha\right)} z_{1}, \ldots, \mathrm{e}^{i\left(\xi_{n-1}+\alpha\right)} z_{n-1}, \mathrm{e}^{i\left(c_{1} \xi_{1}+\ldots+c_{n-1} \xi_{n-1}+\alpha\right)} z_{n}\right) .
$$

Let us now set

$$
\begin{aligned}
\psi_{j} & =\xi_{j}+\alpha, \quad j=1, \ldots, n-1 \\
\psi_{n} & =c_{1} \xi_{1}+\cdots+c_{n-1} \xi_{n-1}+\alpha .
\end{aligned}
$$


Under the condition

$$
c_{1}+\cdots+c_{n-1} \neq 1
$$

these relations define a change of coordinates on the $n$-dimensional torus $\mathbb{T}^{n-1} \times S^{1}$, and in these new coordinates the action can now be written in the very simple fashion

$$
\left(\mathrm{e}^{i \psi_{1}}, \ldots, \mathrm{e}^{i \psi_{n}}\right) \cdot\left(z_{1}, \ldots, z_{n}\right)=\left(\mathrm{e}^{i \psi_{1}} z_{1}, \ldots, \mathrm{e}^{i \psi_{n}} z_{n}\right) .
$$

Notice that under Condition (4.13) the ring of invariant polynomials for this action on $V_{0}$ is generated by the quadratic invariants $\pi_{j}=z_{j} \bar{z}_{j}, j=1, \ldots, n$, and that the strata of this action are obtained by setting some of the $z_{j}$ 's equal to 0 while keeping the others different from 0 . The orbit space for this action can be identified with the positive cone in $\mathbb{R}^{n}\left\{\left(\pi_{1}, \ldots, \pi_{n}\right) / \pi_{j} \geq\right.$ $0, j=1, \ldots, n\}$.

Recall now that in the canonical coordinates introduced in (2.3), the principal part of the reduced bifurcation equation is, by Lemma 3.8, equal to

$$
\begin{aligned}
B\left(v_{0}, \alpha, \lambda, \xi\right)=\left(\lambda \sigma^{\prime}\left(\lambda_{\circ}\right)+\alpha^{2} \nu_{\circ}^{2}\right) v_{0}-\xi^{2} v_{0}- & 2 \alpha \nu_{\circ} \mathbb{J}_{2 n} \xi v_{0}-2 \psi^{\prime}\left(\lambda_{\circ}\right) \nu_{\circ} \alpha \lambda v_{0} \\
& +2 \psi^{\prime}\left(\lambda_{\circ}\right) \lambda \mathbb{J}_{2 n} \xi v_{0}+C\left(v_{0}^{(3)}\right)+\text { h.o.t. }
\end{aligned}
$$

From (4.14) it is clear that the matrices $\mathbb{J}_{2 n}, \mathbb{J}_{2 n} \xi$ and $\xi^{2}$ in (4.15) take, in these newly introduced coordinates, the form:

$$
\begin{gathered}
\mathbb{J}_{2 n}=\left(\begin{array}{ccc}
i & \cdots & 0 \\
\vdots & \ddots & \vdots \\
0 & \cdots & i
\end{array}\right), \quad \mathbb{J}_{2 n} \xi=\left(\begin{array}{cccc}
-\psi_{1} & \cdots & \cdots & 0 \\
\vdots & \ddots & & \vdots \\
\vdots & & -\psi_{n-1} & \vdots \\
0 & \cdots & \cdots & -\psi_{n}
\end{array}\right), \\
\xi^{2}=-\left(\begin{array}{cccc}
\psi_{1}^{2} & \cdots & \cdots & 0 \\
\vdots & \ddots & & \vdots \\
\vdots & & \psi_{n-1}^{2} & \vdots \\
0 & \cdots & \cdots & \psi_{n}^{2}
\end{array}\right) .
\end{gathered}
$$

Using these new coordinates and the $\mathbb{T}^{n-1} \times S^{1}$ equivariance of $B$, we rewrite the components of (4.15) (we omit the complex conjugate part). For $i \in\{1, \ldots, n\}$ we have:

$$
B_{i}(z, \alpha, \lambda, \xi)=\left(\lambda \sigma^{\prime}\left(\lambda_{\circ}\right)+\psi_{i}^{2}+\widehat{C_{i}}\left(\left|z_{1}\right|^{2}, \ldots,\left|z_{n}\right|^{2}\right)+\text { h.o.t. }\right) z_{i}
$$

where

$$
\widehat{C_{i}}\left(\left|z_{1}\right|^{2}, \ldots,\left|z_{n}\right|^{2}\right)=\widehat{c_{i 1}}\left|z_{1}\right|^{2}+\ldots+\widehat{c_{i n}}\left|z_{n}\right|^{2}, \quad \widehat{c_{i j}} \in \mathbb{R}, \quad i, j \in\{1, \ldots, n\},
$$

We can now state a theorem about the bifurcation of RPOs. 
Theorem 4.2 Let $\left(V, \omega, h_{\lambda}\right)$ be a one-parameter family of $G$-Hamiltonian systems that satisfies conditions $(\mathbf{H 1}),(\mathbf{H 2}),(\mathbf{H 3})$, and $\mathbf{( H 4 )}$. Suppose that: (i) the dimension of $V_{0}$ equals twice the rank $n$ of $G \times S^{1}$; (ii) the condition (4.13) on the torus action is satisfied. Then, if the matrix

$$
\Delta=\left(\widehat{c_{n j}}-\widehat{c_{i j}}\right), \quad 1 \leq i \leq n, 1 \leq j \leq n-1,
$$

has maximal rank $n-1$, there exists a family of RPOs with $n$ different frequencies which bifurcates from the trivial solution as $\lambda$ crosses $\lambda_{0}$.

Remark 4.3 The condition on the matrix $\Delta$ is not generic, because the values of the coefficients $\widehat{c_{i j}}$ are constrained by the $G$-equivariance of the operator $C$.

Proof Since we are looking for solutions with $z_{i} \neq 0$, for all $i$, we can factor out $z_{i}$ in each equation $B_{i}=0$. The resulting equations read, for $i \in\{1, \ldots, n\}$,

$$
0=\lambda \sigma^{\prime}\left(\lambda_{\circ}\right)+\psi_{i}^{2}+\widehat{C_{i}}\left(\left|z_{1}\right|^{2}, \ldots,\left|z_{n}\right|^{2}\right)+\text { h.o.t. }
$$

These equations are simply those that we would have obtained by projecting first (4.15) on the orbit space corresponding to the toral action; in all that follows we will denote $\left|z_{i}\right|^{2}$ by $\pi_{i}$. Since by hypothesis (H4), $\sigma^{\prime}\left(\lambda_{\circ}\right) \neq 0$, we can solve any one of these equations for $\lambda$. Let us do so for the equation with $i=n$. By substituting $\lambda$ by the resulting expression in the remaining equations, we have reduced the problem to solving a system of $n-1$ equations which, at leading order, have the form

$$
0=\psi_{i}^{2}-\psi_{n}^{2}+\left(\widehat{c_{i 1}}-\widehat{c_{n 1}}\right) \pi_{1}+\cdots+\left(\widehat{c_{i n}}-\widehat{c_{n n}}\right) \pi_{n}+\text { h.o.t.. }
$$

If the matrix $\Delta$ has maximal rank, we obtain a unique family of solutions of the system (4.16), for which $n-1$ of the $\pi_{i}$ 's depend smoothly on the remaining one and on the parameters $\psi_{j}$, $j=1, \ldots, n$. In order to fix notations, let us assume without loss of generality that we have obtained $\pi_{i}=\pi_{i}\left(\psi_{1}, \ldots, \psi_{n}, \pi_{n}\right)$ for $i=1, \ldots, n-1$. These solutions still have to lie inside the orbit space, that is, we still have to check the additional conditions $\pi_{i} \geq 0$. However, since the $\psi_{j}$ 's are free parameters, the quantities $\psi_{i}^{2}-\psi_{n}^{2}$ can take any real value. Therefore, if we set $z_{n}=0$, we can always find values for the $\psi_{j}$ 's such that $\pi_{i}>0$ for $i=1, \ldots, n-1$. These inequalities are still satisfied if the $\psi_{j}$ 's are close enough to these values and $\pi_{n}>0$ is close enough to 0. Finally, since these solutions lie on the principal stratum for the action of $\mathbb{T}^{n-1} \times S^{1}$, the corresponding RPOs have $n$ different frequencies which depend smoothly on $\lambda$.

Remark 4.4 In the problem with $O(2)$ symmetry analyzed in Section 4.2 , we have $n=2$, $c_{1}=-1, \widehat{c_{11}}=\widehat{c_{22}}$ and $\widehat{c_{12}}=\widehat{c_{21}}$. The hypotheses of Theorem 4.2 are therefore generically satisfied in this case.

Remark 4.5 As it was already the case with Theorem 3.3, Theorem 4.2 still applies if instead of $G \times S^{1}$ acting in $V_{0}$, we consider the group $N(H) / H$ acting in $V_{0}^{H}$, where $H$ is an isotropy subgroup of the $G \times S^{1}$-action. In the next section we shall see an application of this remark. 


\subsection{Hamiltonian Hopf bifurcation with $S O(3)$ symmetry}

Hopf bifurcation problems with $S O(3)$ symmetry for dissipative systems have been investigated by several authors in the case in which the eigenspaces associated with the critical eigenvalues is the direct sum of twice the five dimensional (real) irreducible representation of $S O(3)$ (see [GoSt85]), [oRo89], MRS88, and Le97]). This is the simplest possible case with $S O(3)$ symmetry which does not reduce to Hopf bifurcation with either trivial or $O(2)$ symmetry. Nevertheless, it leads to a normal form in a ten dimensional real vector space. The list of solutions with maximal isotropy, hence purely periodic ones, has been given in GoSt85] and in MRS88. However, the most interesting feature of this problem is the possibility of having a bifurcated branch of RPOs in a six dimensional subspace. This was first found by [oRo89]. Another approach was taken by [Le97] (using orbit space reduction) who did not recover the result of [oRo89. This remark shows the level of difficulty found in obtaining direct branching of RPOs via Hopf bifurcation for equivariant vector fields. In the Hamiltonian context, a related work by Haaf, Roberts and Stewart HRS92 has shown the existence of families of periodic orbits with maximal isotropy for a Hamiltonian in $\mathbb{R}^{10}$ which is invariant under the same $S O(3)$-action.

In the sequel we investigate the Hamiltonian Hopf bifurcation with $S O(3)$ symmetry, when the subspaces $V_{0}$ and $V_{1}$ are associated with this ten dimensional representation and we shall see that, in this case, Theorem 4.2 applies and shows the existence of several families of RPOs.

Let

$$
V=V_{0} \oplus V_{1} \simeq \mathbb{R}^{10}
$$

We identify $\mathbb{R}^{10}$ with $\mathbb{R}^{5} \otimes \mathbb{C}$ and consider the action of $S O(3)$ on $\mathbb{R}^{5}$ given by its irreducible representation on the space of spherical harmonics of degree 2. Equivalently, we may identify $\mathbb{R}^{5}$ with the space $W$ of $3 \times 3$ real symmetric matrices with trace 0 , and consider the action of $S O(3)$ on $W$ defined by

$$
\rho_{A}(M)=A^{-1} M A, \quad A \in S O(3), M \in W .
$$

This definition extends naturally to $\mathbb{R}^{5} \otimes \mathbb{C}$ with the same formula, $M$ now having complex coefficients. We shall therefore identify in all that follows $V_{0}$ with $W \otimes \mathbb{C}$. The $S^{1}$-action on $V_{0}$ is simply defined as multiplication by $e^{i \theta}$ in $\mathbb{C}$, that is, $\theta \cdot M:=e^{i \theta} M$.

Any $M \in W \otimes \mathbb{C}$ decomposes uniquely as

$$
M=\sum_{m=-2}^{2} z_{m} B_{m}
$$


where

$$
\begin{aligned}
B_{0} & =\left(\begin{array}{lll}
1 & 0 & 0 \\
0 & 1 & 0 \\
0 & 0 & 2
\end{array}\right), B_{1}=\left(\begin{array}{lll}
0 & 0 & 1 \\
0 & 0 & i \\
1 & i & 0
\end{array}\right), B_{-1}=\bar{B}_{1} \\
B_{2} & =\left(\begin{array}{ccc}
1 & i & 0 \\
i & -1 & 0 \\
0 & 0 & 0
\end{array}\right), B_{-2}=\bar{B}_{2} .
\end{aligned}
$$

We now list the isotropy types for the action of $G=S O(3) \times S^{1}$ on $V_{0}$ that we have just defined. We use the presentation and results of [HRS92]. Figure 4.2 shows the isotropy lattice of the $G$-action and the dimension of the corresponding fixed-point subspaces.

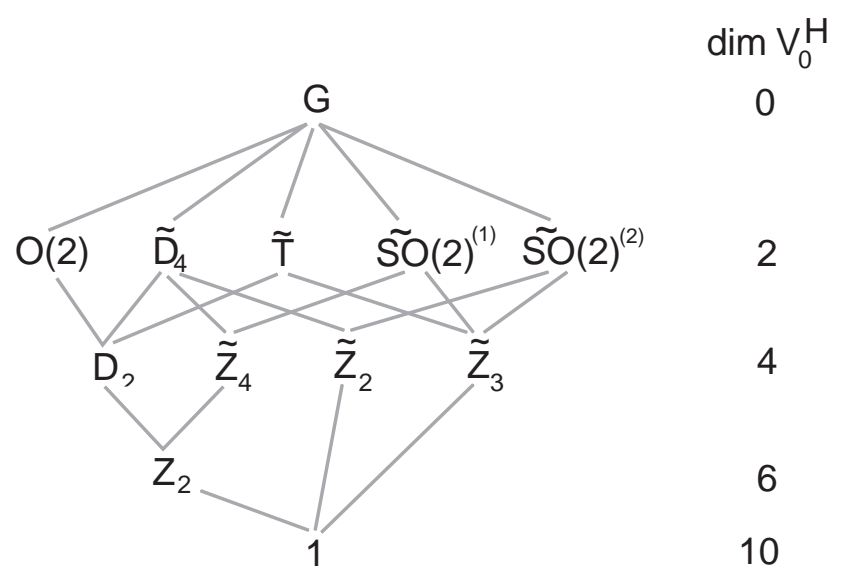

Figure 4.2: Isotropy lattice of the $S O(3) \times S^{1}$-action.

Notations: $\tilde{H}$ is a subgroup isomorphic to $H \subset O(3)$ but such that $\tilde{H} \cap S^{1} \neq 1$ (here 1 is the trivial group). In particular, $\tilde{\mathbb{Z}}_{n}$ is the group generated by $\left(R_{n},-2 \pi / n\right) \in S O(3) \times S^{1}$, where $R_{n}$ is a rotation of angle $2 \pi / n$.

By Corollary 3.9, for any subgroup $H$ with $\operatorname{dim}\left(V_{0}^{H}\right)=2$ there exists a branch of periodic solutions having this symmetry. Let us now consider those isotropy subgroups having a fixedpoint subspace of dimension 4. The largest subgroup acting faithfully in $V_{0}^{H}$ is $N(H) / H$. We list below the different $N(H) / H$ for the non maximal isotropy subgroups:

1. $N\left(D_{2}\right) / D_{2} \simeq D_{3} \times S^{1}$;

2. $N\left(\tilde{\mathbb{Z}}_{4}\right) / \tilde{\mathbb{Z}}_{4} \simeq N\left(\tilde{\mathbb{Z}}_{2}\right) / \tilde{\mathbb{Z}}_{2} \simeq O(2) \times S^{1}$;

3. $N\left(\tilde{\mathbb{Z}}_{3}\right) / \tilde{\mathbb{Z}}_{3} \simeq S O(2) \times S^{1}$;

4. $N\left(\mathbb{Z}_{2}\right) / \mathbb{Z}_{2} \simeq O(2) \times S^{1}$; 
5. $N(1)=G$.

In Case 1 we see that solutions with isotropy $D_{2}$ are always periodic. Case 2 corresponds to the problem described in Section 4.2 (Hopf bifurcation with $O(2)$ symmetry). It was noticed in HRS92] that the equations in $V_{0}^{H}$ do not degenerate despite the fact that they come from a system with higher symmetry, which leads us to conclude that families of RPOs with two frequencies and with spatio-temporal symmetry $\tilde{\mathbb{Z}}_{4}$ as well as $\tilde{\mathbb{Z}}_{2}$ do generically bifurcate. Case 3 falls in the framework of Section 4.3, since the symmetry group is $S O(2) \times S^{1}$. However, because the equations in $V_{0}^{\tilde{\mathbb{Z}}_{3}}$ are the restriction in that subspace of a system with higher symmetry in $V_{0}$, we need to compute the cubic order terms in order to insure that no "hidden" degeneracy occurs. We use the argument proved in [HRS92]; we can choose $\tilde{\mathbb{Z}}_{3}$ so that, by introducing complex coordinates,

$$
V_{0}^{\tilde{\mathbb{Z}}_{3}}=\left\{z_{1} B_{1}+z_{2} B_{-2},\left(z_{1}, z_{2}\right) \in \mathbb{C}^{2}\right\}
$$

and the action of $S O(2)$ is then defined by

$$
\phi \cdot(w, z)=\left(e^{i \phi}, e^{-2 i \phi}\right) .
$$

With the notations of Section 4.3, we therefore have

$$
\psi_{1}=\phi \text { and } c_{1}=-2
$$

The expression for the cubic $G$-equivariant terms is

$$
C\left(M^{(3)}\right)=b_{1} \operatorname{tr}(M \bar{M}) M+b_{2} \operatorname{tr}\left(M^{2}\right) \bar{M}+b_{3}\left(M^{2} \bar{M}+\bar{M} M^{2}-\frac{2}{3} \operatorname{tr}\left(M^{2} \bar{M}\right) I d\right)
$$

with $b_{j}$ real coefficients depending on the specific Hamiltonian at hand. Setting $M=z_{1} B_{1}+$ $z_{2} B_{-2}$ we obtain after calculation in $V_{0}^{\tilde{\mathbb{Z}}_{3}}$ the expression

$$
C\left(M^{(3)}\right)=4\left(\left(b_{1}+\frac{b_{3}}{2}\right)\left|z_{1}\right|^{2}+\left(b_{1}+b_{3}\right)\left|z_{2}\right|^{2}\right) z_{1} B_{1}+4\left(\left(b_{1}+b_{3}\right)\left|z_{1}\right|^{2}+b_{1}\left|z_{2}\right|^{2}\right) z_{2} B_{-2} .
$$

Let us now check whether the $1 \times 2$ matrix $\Delta$ of Theorem 4.2 has maximal rank. From the above expression we deduce that

$$
\widehat{c_{11}}-\widehat{c_{21}}=-2 b_{3}, \widehat{c_{12}}-\widehat{c_{22}}=4 b_{3} .
$$

Therefore the maximality hypothesis is satisfied iff $b_{3} \neq 0$ (which is a generic condition). Cases 4 and 5 are beyond the range of applicability of Theorem 4.2 . 


\section{Appendix}

\subsection{On the invariance properties of the resonance subspace}

In what follows we will sketch the proof of some of the facts about the invariance properties of the resonance subspace mentioned in the preliminaries section when $(V, \omega)$ is a symplectic representation space of the Lie group $G$ and the Hamiltonian vector field $A$ is $G$-equivariant.

The resonance subspace $U_{\nu_{\circ}}$ is $G$-invariant Let $A=A_{s}+A_{n}$ be the Jordan-Chevalley decomposition of $A$. Since by hypothesis $A$ is $G$-equivariant, if $\Phi: G \times V \rightarrow V$ denotes the $G$ action, for any $g \in G$, we have that $\Phi_{g} A=A \Phi_{g}$. Equivalently, $\Phi_{g} A_{s}+\Phi_{g} A_{n}=A_{s} \Phi_{g}+A_{n} \Phi_{g}$, and hence $\Phi_{g} A_{n} \Phi_{g^{-1}}+\Phi_{g} A_{s} \Phi_{g^{-1}}=A_{n}+A_{s}$. Since $\Phi_{g} A_{n} \Phi_{g^{-1}}$ is nilpotent, $\Phi_{g} A_{s} \Phi_{g^{-1}}$ is semisimple, $\left[\Phi_{g} A_{n} \Phi_{g^{-1}}, \Phi_{g} A_{s} \Phi_{g^{-1}}\right]=0$, and the Jordan-Chevalley decomposition is unique, we have that

$$
\Phi_{g} A_{n} \Phi_{g^{-1}}=A_{n} \quad \text { and } \quad \Phi_{g} A_{s} \Phi_{g^{-1}}=A_{s}
$$

necessarily. This implies the $G$-invariance of $U_{\nu_{\circ}}=\operatorname{ker}\left(e^{A_{s} T_{\nu_{\circ}}}-I\right)$. Indeed, let $v \in U_{\nu_{\circ}}$. Hence, $e^{A_{s} T_{\nu_{\circ}} v}=v$. At the same time, for any $g \in G$,

$$
e^{A_{s} T_{\nu_{\circ}}}\left(\Phi_{g} v\right)=\Phi_{g} e^{A_{s} T_{\nu_{\circ}}} v=\Phi_{g} v
$$

hence $\Phi_{g} v \in U_{\nu_{\circ}}$, that is, $U_{\nu_{\circ}}$ is $G$-invariant.

The $S^{1}$-action and the $G$-action on $U_{\nu_{\circ}}$ commute Let $\Psi: S^{1} \times U_{\nu_{\circ}} \rightarrow U_{\nu_{\circ}}$ be the $S^{1}$-action on $U_{\nu_{\circ}}$. For any $g \in G$ and any $\theta \in S^{1}$ :

$$
\Phi_{g} \Psi_{\theta}=\Phi_{g} e^{\theta A_{s}}=e^{\theta A_{s}} \Phi_{g}=\Psi_{\theta} \Phi_{g},
$$

as required.

\subsection{Proof of Lemma 3.7}

The defining relation (3.4) of the function $v_{1}$ implies that for any $v_{0} \in V_{0}, \alpha, \lambda \in \mathbb{R}$, and $\xi \in \mathfrak{h}$ we have that

$$
(\mathbb{I}-\mathbb{P}) \nabla_{U_{\nu_{\mathrm{o}}}}\left(\widehat{h_{\lambda}}-\mathbf{J}^{1+\alpha}-\mathbf{K}^{\xi}\right)\left(v_{0}+v_{1}\left(v_{0}, \alpha, \lambda, \xi\right)\right)=0 .
$$

Consequently, for any $w_{1} \in V_{1}$ :

$$
\begin{aligned}
0 & =\left.\frac{d}{d t}\right|_{t=0}\left\langle\nabla_{U_{\nu_{\mathrm{o}}}}\left(\widehat{h_{\lambda}}-\mathbf{J}^{1+\alpha}-\mathbf{K}^{\xi}\right)\left(t v_{0}+v_{1}\left(t v_{0}, \alpha, \lambda, \xi\right)\right), w_{1}\right\rangle \\
& =\left.\frac{d}{d t}\right|_{t=0} \mathbf{d}\left(\widehat{h_{\lambda}}-\mathbf{J}^{1+\alpha}-\mathbf{K}^{\xi}\right)\left(t v_{0}+v_{1}\left(t v_{0}, \alpha, \lambda, \xi\right)\right) \cdot w_{1} \\
& =\mathbf{d}^{2}\left(\widehat{h_{\lambda}}-\mathbf{J}^{1+\alpha}-\mathbf{K}^{\xi}\right)(0)\left(v_{0}+D_{V_{0}} v_{1}(0, \alpha, \lambda, \xi) \cdot v_{0}, w_{1}\right) .
\end{aligned}
$$


If we use (2.6) and (2.15), the previous expression can be matricially expressed as

$$
\begin{aligned}
0= & \left(0, w_{1}\right) \cdot\left(\begin{array}{cc}
\sigma(\lambda) \mathbb{I}_{2 n} & \tau(\lambda) \mathbb{I}_{2 n}+(\psi(\lambda)-(1+\alpha)) \mathbb{J}_{2 n}-\xi \\
\tau(\lambda) \mathbb{I}_{2 n}-(\psi(\lambda)-(1+\alpha)) \mathbb{J}_{2 n}+\xi & \rho(\lambda) \mathbb{I}_{2 n}
\end{array}\right) \\
& \cdot\left(\begin{array}{c}
v_{0} \\
D_{V_{0}} v_{1}(0, \alpha, \lambda, \xi) \cdot v_{0}
\end{array}\right) \\
= & w_{1}^{T}\left[\tau(\lambda) \mathbb{I}_{2 n}-(\psi(\lambda)-(1+\alpha)) \mathbb{J}_{2 n}+\xi+\rho(\lambda) D_{V_{0}} v_{1}(0, \alpha, \lambda, \xi)\right] v_{0} .
\end{aligned}
$$

Given that the previous equation is valid for no matter what $v_{0} \in V_{0}$ and $w_{1} \in V_{1}$, we can conclude that

$$
D_{V_{0}} v_{1}(0, \alpha, \lambda, \xi)=\frac{\psi(\lambda)-(1+\alpha)}{\rho(\lambda)} \mathbb{J}_{2 n}-\frac{\tau(\lambda)}{\rho(\lambda)} \mathbb{I}_{2 n}-\frac{\xi}{\rho(\lambda)}
$$

as required.

Acknowledgments. We thank M. Dellnitz and I. Melbourne for their help and patience concerning our questions on their equivariant Williamson normal form MD93. We also thank A. Vanderbauwhede for his valuable help when we were in the process of understanding his paper VvdM95. Thanks also go to D. Burghelea, M. Field, V. Ginzburg, A. Hernández, K. Hess, J. E. Marsden, and J. Montaldi for their assistance at various points in the development of this work.

\section{References}

[AM78] Abraham, R., and Marsden, J.E. [1978] Foundations of Mechanics. Second edition, Addison-Wesley.

[AMR99] Abraham, R., Marsden, J.E., and Ratiu, T.S. [1988] Manifolds, Tensor Analysis, and Applications. Volume 75 of Applied Mathematical Sciences, Springer-Verlag.

[Ba94] Bartsch, T. [1994] Topological Methods for Variational Problems with Symmetries. Springer Lecture Notes in Mathematics, vol. 1560.

[Bott82] Bott, R. [1982] Lectures on Morse Theory, old and new. Bull. Amer. Math. Soc., $7(2): 331-358$.

[BT82] Bott, R. and Tu, L. [1982] Differential Forms in Algebraic Topology. Graduate Texts in Mathematics, vol. 82. Springer-Verlag.

[Bre72] Bredon, G.E. [1972] Introduction to Compact Transformation Groups. Academic Press. 
[Bri90] Bridges, T. J. [1990] Bifurcation of periodic solutions near a collision of eigenvalues of opposite signature. Math. Proc. Camb. Phil. Soc., 108:575-601.

[Bri90a] Bridges, T. J. [1990] The Hopf bifurcation with symmetry for the Navier-Stokes equation in $\left(L_{p}(\Omega)\right)^{n}$ with application to plane Poiseuille flow.Arch. Rational Mech. Anal., 106:335-376.

[BrL75] Bröcker, Th., and Lander, L. [1975] Differentiable germs and catastrophes. London Mathematical Society Lecture Note Series, volume 17. Cambridge University Press.

[CKM95] Chossat, P., Koenig, M., and Montaldi, J. [1995] Bifurcation générique d'ondes d'isotropie maximale. C. R. Acad. Sci. Paris Sér. I Math., 320:25-30.

[ChL00] Chossat, P. and Lauterbach, L. [2000] Methods in Equivariant bifurcations and Dynamical Systems. Advanced Ser. in Nonlinear Dyn. 15, World Scientific.

[CLOR99] Chossat, P., Lewis, D., Ortega, J.-P., and Ratiu, T. S. [1999] Bifurcation of relative equilibria in mechanical systems with symmetry. Preprint.

[CP86] Clapp, M., and Puppe, D. [1986] Invariants of Lusternik-Schnirelmann type and the topology of critical sets. Transactions Amer. Math. Soc., 298:603-620.

[CP91] Clapp, M., and Puppe, D. [1991] Critical point theory with symmetries. J. reine. angew. Math., 418:1-29.

[DMM92] Dellnitz, M., Melbourne, I., and Marsden, J. E. [1992] Generic Bifurcation of Hamiltonian vector fields with symmetry. Nonlinearity, 5:979-996.

[Fa85] Fadell, E. [1985] The equivariant Lusternik-Schnirelmann method for invariant functionals and relative cohomologica index theories. In Méthodes topologiques en analyse non linéaire. A. Granas (ed.) Sémin. Math. Sup. No. 95. Montréal, 41-70.

[FiRi89] Field, M. J. and Richardson, R. W. [1989] Symmetry breaking and the maximal isotropy subgroup conjecture for reflection groups. Arch. Rational Mech. Anal., 105:61-94.

[Fi94] Field, M. [1996] Symmetry breaking for compact Lie groups, Memoirs of the American Math. Soc. 120.

[Fied88] Fiedler, B. [1988] Global Bifurcation of Periodic Solutions with Symmetry. Lecture Notes in Mathematics, vol. 1309. Springer-Verlag.

[G83] Golubitsky, M. [1983] The Bénard Problem, symmetry, and the lattice of isotropy subgroups. In Bifurcation Theory, Mechanics, and Physics. Bruter, C. P. et al. (eds.), pages 225-256. Reidel. 
[GMSD95] Golubitsky, M., Marsden, J. E., Stewart, I., and Dellnitz, M. [1995] The constrained Liapunov-Schmidt procedure and periodic orbits. In Normal Forms and Homoclinic Chaos, pages 81-127. Langford, W. F. and Nagata, W. eds. Fields Institute Communications, 4 .

[GoS85] Golubitsky, M., and Schaeffer, D.G. [1985] Singularities and Groups in Bifurcation Theory: Vol. I. Applied Mathematical Sciences, Vol. 51, Springer-Verlag.

[GoSt85] Golubitsky, M. and Stewart, I. [1985] Hopf bifurcation in the presence of symmetry. Arch. Rational Mech. Anal., 87:107-165.

[GoS87] Golubitsky, M. and Stewart, I. With an appendix by J. E. Marsden. [1987] Generic bifurcation of Hamiltonian systems with symmetry. Physica D, 24:391-405.

[GSS88] Golubitsky, M., Stewart, I., and Schaeffer, D.G. [1988] Singularities and Groups in Bifurcation Theory: Vol. II. Applied Mathematical Sciences, Vol. 69, SpringerVerlag.

[GS84] Guillemin, V. and Sternberg, S. [1984] Symplectic Techniques in Physics. Cambridge University Press.

[HRS92] Haaf, H., Roberts, M., Stewart, I. A Hopf bifurcation with spherical symmetry. ZAMP, 43: 793-826.

[Hal74] Halmos, P. R. [1974] Finite-dimensional Vector Spaces. Springer-Verlag.

[Hopf26] Hopf, H. [1926] Vektorfelder in n-dimensionalen Manningfaltigkeiten. Math. Ann., 96:225-250.

[Hu72] Humphreys, J. E. [1972] Introduction to Lie Algebras and Representation Theory. Graduate Texts in Mathematics, no. 9. Springer-Verlag.

[IoRo89] Iooss, G., Rossi, M. [1989] Hopf bifurcation in the presence of spherical symmetry: analytical results. SIAM J. Math. Anal., 20, 3:511-532.

[J80] Jiang, B. [1980] Lectures on Nielsen Fixed Point Theory. Contemporary Mathematics, vol. 14. American Mathematical Society.

[Kaw91] Kawakubo, K. [1991] The Theory of Transformation Groups. Oxford University Press.

[KMS96] Kirk, V., Marsden, J.E., and Silber, M. [1996] Branches of stable three-tori using Hamiltonian methods in Hopf bifurcation on a rhombic lattice. Dynamics and Stability of Systems, 11(4):267-302.

[Mar89] Marzantowicz, W. [1989] A G-Lusternik-Schnirelamn category of space with an action of a compact Lie group. Topology, 28:403-412. 
[Koe95] Koenig, M. [1995] Une Exploration des Espaces d'Orbites des Groupes de Lie Compacts et de leurs Applications à l'Étude des Bifurcations avec Symétrie. Ph. D. Thesis. Institut Non Linéaire de Nice. November 1995.

[Le97] Leis, C. [1997] Hopf bifurcations in systems with spherical symmetry, part I. Documenta Mathematica, 2:61-113.

[MD93] Melbourne, I., and Dellnitz, M. [1993] Normal forms for linear Hamiltonian vector fields commuting with the action of a compact Lie group. Math. Proc. Camb. Phil. Soc., 114:235-268.

[Mey86] Meyer, K. R. [1986] Bibliographical notes on generic bifurcation in Hamiltonian systems. In Multiparameter Bifurcation Theory, Contemp. Math. no. 56 (American Mathematical Society), pp. 373-381.

[MeyS71] Meyer, K. R. and Schmidt, D. S. [1971] Periodic orbits near $L_{4}$ for mass ratios near the critical mass ratio of Routh. Celestial Mech., 99-109.

[MRS88] Montaldi, J.A., Roberts, R.M., and Stewart, I.N. [1988] Periodic solutions near equilibria of symmetric Hamiltonian systems. Phil. Trans. R. Soc. Lond. A, 325:237-293.

[M76] Moser, J. [1976] Periodic orbits near an equilibrium and a theorem by Alan Weinstein. Comm. Pure Appl. Math., 29:727-747.

[Po76] Poènaru, V. [1976] Singularités $C^{\infty}$ en présence de symétrie. Lecture Notes in Mathematics, volume 510. Springer-Verlag.

[Sch69] Schwartz, J. T. [1969] Nonlinear Functional Analysis. Gordon and Breach.

[VvdM95] Vanderbauwhede, A. and van der Meer, J. C. [1995] General reduction method for periodic solutions near equilibria. In Normal Forms and Homoclinic Chaos, pages 273-294. Langford, W. F. and Nagata, W. eds. Fields Institute Communications, 4.

[vdM85] van der Meer, J. C. [1985] The Hamiltonian Hopf Bifurcation. Lecture Notes in Mathematics, 1160. Springer Verlag.

[vdM90] van der Meer, J. C. [1990] Hamiltonian Hopf bifurcation with symmetry. Nonlinearity, 3:1041-1056.

[vdM96] van der Meer, J. C. [1996] Degenerate Hamiltonian Hopf bifurcations. In Conservative Systems and Quantum Chaos, pages 159-176. Bates, L. M. and Rod, D. L., eds. Fields Institute Communications, 8. 
[W73] Weinstein, A. [1973] Normal forms for nonlinear Hamiltonian systems. Inventiones Math., 20:47-57.

[W77] Weinstein, A. [1977] Symplectic $V$-manifolds, periodic orbits of Hamiltonian systems, and the volume of certain Riemannian manifolds. Comm. Pure Appl. Math., $30: 265-271$.

[Wil36] Williamson, J. [1936] On the algebraic problem concerning the normal forms of linear dynamical systems. Amer. J. Math., 58:141-163. 